\title{
THREE WIDE PLANETARY-MASS COMPANIONS TO FW TAU, ROXs 12, AND ROXs 42B
}

\author{
Adam L. Kraus ${ }^{1,2}$, Michael J. Ireland ${ }^{3,4}$, Lucas A. Cieza ${ }^{5,6}$, Sasha Hinkley ${ }^{7}$, \\ Trent J. Dupur ${ }^{2}$, Brendan P. Bowler ${ }^{5,7}$, And Michael C. LiU ${ }^{5}$ \\ ${ }^{1}$ Department of Astronomy, The University of Texas at Austin, Austin, TX 78712, USA \\ ${ }^{2}$ Harvard-Smithsonian Center for Astrophysics, 60 Garden St., Cambridge, MA 02138, USA \\ ${ }^{3}$ Department of Physics and Astronomy, Macquarie University, NSW 2109, Australia \\ ${ }^{4}$ Australian Astronomical Observatory, P.O. Box 296, Epping NSW 1710, Australia \\ ${ }^{5}$ Institute for Astronomy, University of Hawaii, 2680 Woodlawn Dr., Honolulu, HI 96822, USA \\ ${ }^{6}$ Universidad Diego Portales, Facultad de Ingeniera, Av. Ejército 441, Santiago, Chile \\ ${ }^{7}$ Department of Astronomy, California Institute of Technology, 1200 E. California Blvd., MC 249-17, Pasadena, CA 91125, USA \\ Received 2012 December 21; accepted 2013 November 28; published 2013 December 31
}

\begin{abstract}
We report the discovery of three planetary-mass companions $\left(M=6-20 M_{\text {Jup }}\right)$ in wide orbits $(\rho \sim 150-300 \mathrm{AU})$ around the young stars FW Tau (Taurus-Auriga), ROXs 12 (Ophiuchus), and ROXs 42B (Ophiuchus). All three wide planetary-mass companions (PMCs) were reported as candidate companions in previous binary survey programs, but then were neglected for $>10 \mathrm{yr}$. We therefore obtained followup observations that demonstrate that each candidate is comoving with its host star. Based on the absolute $M_{K^{\prime}}$ magnitudes, we infer masses (from hot-start evolutionary models) and projected separations of $10 \pm 4 M_{\text {Jup }}$ and $330 \pm 30 \mathrm{AU}$ for FW Tau b, $16 \pm 4 M_{\text {Jup }}$ and $210 \pm 20 \mathrm{AU}$ for ROXs 12 , and $10 \pm 4 M_{\mathrm{Jup}}$ and $140 \pm 10 \mathrm{AU}$ for ROXs $42 \mathrm{~B}$ b. We also present similar observations for 10 other candidates that show that they are unassociated field stars, as well as multicolor $J H K^{\prime} L^{\prime}$ near-infrared photometry for our new PMCs and for five previously identified substellar or planetary-mass companions. The near-infrared photometry for our sample of eight known and new companions generally parallels the properties of free-floating, low-mass brown dwarfs in these star-forming regions. However, five of the seven objects with $M<30 M_{\text {Jup }}$ are redder in $K^{\prime}-L^{\prime}$ than the distribution of young free-floating counterparts of similar $J-K^{\prime}$ color. We speculate that this distinction could indicate a structural difference in circumplanetary disks, perhaps tied to higher disk mass since at least two of the objects in our sample are known to be accreting more vigorously than typical free-floating counterparts.
\end{abstract}

Key words: brown dwarfs - planetary systems - planets and satellites: formation - planets and satellites: gaseous planets - stars: individual (FW Tau, ROXs 12, ROXs 42B) - stars: pre-main sequence

Online-only material: color figures

\section{INTRODUCTION}

Over the past decade, direct imaging surveys for extrasolar planets have discovered a small number of planetary-mass companions (hereafter called PMCs; $\lesssim 20 M_{\text {Jup }}$ ) at $\gtrsim 50 \mathrm{AU}$ orbital radii from young stars in nearby star-forming regions. The prototypical wide PMC, 2M1207-3933 b, consists of a 4-8 $M_{\text {Jup }}$ companion located $\rho \sim 40$ AU away from a $35 M_{\text {Jup }}$ brown dwarf (BD; Chauvin et al. 2004). Since its discovery, $\sim 10$ other PMCs have been found, most of which orbit highermass primaries $\left(\sim 0.3-2.0 M_{\odot}\right.$; Neuhäuser et al. 2005; Luhman et al. 2006; Lafrenière et al. 2008; Schmidt et al. 2008; Ireland et al. 2011; Delorme et al. 2013; Bowler et al. 2013). Recent exoplanet discoveries like HR 8799 bcde, GJ 504 b, and HD 95086 b (Marois et al. 2008; Kuzuhara et al. 2013; Rameau et al. 2013) suggest that giant planets can form at moderately wide orbital radii $(\sim 50-100 \mathrm{AU})$. However, there are confirmed PMCs with orbits as wide as $300 \mathrm{AU}$ (i.e., 1RXSJ1609 b and GSC 6214-210 b; Lafrenière et al. 2008; Ireland et al. 2011).

These wide-separation PMCs pose a significant challenge to existing models of star and planet formation. Their orbital radii are so large that it is unlikely that they could form like traditional planetary systems, as the classical core accretion timescale is far too long ( $\gg 100 \mathrm{Myr}$ at $a>100 \mathrm{AU}$; Pollack et al. 1996) and Class II disks (Andrews \& Williams 2005, 2007) should not become Toomre unstable to direct fragmentation of $\sim 5 M_{\text {Jup }}$ objects at these extreme radii (Dodson-Robinson et al.
2009; Meru \& Bate 2010; Kratter \& Murray-Clay 2011, and references therein). However, it is equally unlikely that PMCs could form like binary companions during the Class 0/I stages, since they fall near or below the opacity-limited minimum mass (Bate 2005) and should accrete to become stellar or BD binary companions unless they form at nearly the same time that the circumstellar envelope is exhausted (Kratter et al. 2010). Nonetheless, it appears that one of these outcomes must occur.

We previously conducted a combined analysis of many highresolution imaging surveys of young stars (Kraus et al. 2014) that demonstrated that very low-mass companions occur at a frequency of $\gtrsim 1 \%-2 \%$ for orbital radii of 75-750 AU and masses of 2-40 $M_{\text {Jup }}$. Given this non-trivial frequency, it appears that they represent a genuine population. However, there are still only $\sim 10$ PMCs known in star-forming regions and only two were discovered in statistically robust surveys (Ireland et al. 2011). Most such PMCs were discovered serendipitously or in unpublished surveys that cannot be used for calculating population statistics, so the demographics (frequency, mass function, and semi-major axis distribution) are highly uncertain. Some robust surveys now have been conducted in older populations (Chauvin et al. 2010; Vigan et al. 2012; Rameau et al. 2012; Biller et al. 2013; Nielsen et al. 2013), but uncertainties in the ages and dynamical birth environments make their interpretation more complex.

Even in the absence of population statistics, detailed studies of individual PMCs provide important evidence regarding their 
formation and early evolution. Spectroscopic studies of their atmospheres have shown that PMCs resemble free-floating BDs with late-M and early-L spectral types (Lafrenière et al. 2008; Patience et al. 2010; Bonnefoy et al. 2010; Bowler et al. 2011; Faherty et al. 2013; Allers \& Liu 2013) and hence they do not show evidence for metallicity enhancement or otherwise non-solar bulk compositions (e.g., Madhusudhan et al. 2011). Furthermore, Bowler et al. (2011) demonstrated that GSC 6214$210 \mathrm{~b}$ has extraordinarily strong $\mathrm{Pa} \beta$ emission, a strong indicator for vigorous accretion from a circumsubstellar disk; the survival of this disk places strong constraints on the past dynamical history, arguing that the PMC formed in situ and was not ejected out to its current position. Other PMCs also show evidence of possible $\mathrm{Pa} \beta$ emission, including GQ Lup b (Seifahrt et al. 2007) and CT Cha b (Schmidt et al. 2008), and 1RXSJ1609 might have an unresolved (system) excess at $24 \mu \mathrm{m}$ (Bailey et al. 2013). These results indicate that accretion disks could be common; we return to this topic in our discussion of the companion to FW Tau. Finally, partial orbital arcs, such as the existing measurement for GQ Lup b (Neuhäuser et al. 2008), also can provide evidence for the formation site and orbital evolution of PMCs.

The discovery of additional PMCs will be crucial in mapping the mass and separation distributions and in enabling more of these detailed studies. In this paper, we present the identification of three PMCs $\left(M=5-20 M_{\text {Jup }}\right)$ in wide orbits ( $\rho \sim 150-300 \mathrm{AU})$ around young stars $(\tau \sim 1-3 \mathrm{Myr})$. All were identified as candidate binary companions $>10 \mathrm{yr}$ ago, but subsequently ignored in the literature. We also confirm that 10 additional candidates are unassociated field stars and present multicolor data for our new discoveries and for five previously identified PMCs. In Section 2, we describe our sample of candidate companions, summarize any previous observations of them, and establish the properties of the host stars. In Section 3, we describe our new observations and how we derived the properties of each candidate. In Section 4, we discuss the detailed properties of three newly identified companions, summarize the identification of 10 candidates as unassociated field stars, and discuss our observations of known PMCs. Finally, in Section 5, we use the combined multicolor data to compare the colors and luminosities of the eight PMCs (three new and five known) to free-floating young BDs and theoretical models.

\section{THE SAMPLE}

\subsection{Candidate Wide PMCs}

We list the targets considered in this study in Table 1. Five of our targets (ROXs 12, ROXs 42B, ${ }^{8}$ DoAr 22, PDS 70, and FW Tau) were identified as candidate companions during the years 2001-2005, but then were subsequently neglected. Ratzka et al. (2005) conducted a $K$-band speckle imaging survey for stellar binarity in the Ophiuchus star-forming region and, by analyzing their data with shift-and-add stacking, they also identified faint candidate companions to ROXs 12, ROXs 42B $\mathrm{A}+\mathrm{B}$, and DoAr 22. During our own observations, we also found an additional faint candidate companion to ROXs 42B that was located interior to the published candidate. Riaud et al. (2006) also discovered a candidate companion to PDS 70, a member of Upper Centaurus-Lupus, while conducting a $K$-band

\footnotetext{
8 Regarding nomenclature, we note that the "B" in ROXs 42B indicates that it is the second brightest optical counterpart in the error circle for the X-ray source ROXs 42. It does not denote anything regarding the binarity of the system (which is unlikely to be associated with ROXs 42A or ROXs 42C).
}

coronagraphic adaptive optics (AO) imaging survey of young southern stars. Finally, White \& Ghez (2001) discovered a wide candidate companion to FW Tau A+B while obtaining broadband optical colors of known young binary systems in Taurus-Auriga with the Hubble Space Telescope (HST); the candidate was represented by marginal (and red) detections at $R_{C}$ and $I_{C}$, plus a significant detection in the narrowband $H \alpha$ filter, indicating that it had significant $H \alpha$ emission.

Another seven of our targets (2M04153916, HD 27659, ScoPMS 42b, GSC 06191-00019, GSC 06793-00994, GSC 06794-00156, and UScoJ1608-1935) were identified to have candidate companions in the course of our own survey programs. Two of these stars (GSC06793-00994 and GSC 0679400156) were part of the sample of Upper Scorpius (Upper Sco) members we described in Ireland et al. (2011), but their candidate companions could not be tested for association in that work. Another three stars (GSC 06191-00019, ScoPMS 42b, and UScoJ1608-1935) were observed to have faint candidate companions during the same campaign, but we did not include them in our previous analysis because they are binary systems with projected separations that impinge on the PMC semi-major axis range ( $\rho>150$ mas). Finally, two targets (2M04153916 and HD 27659) were identified to have faint candidate companions during a snapshot $K^{\prime}$ band AO imaging of new Taurus members recently discovered by Luhman et al. (2009) and Rebull et al. (2010); these observations were primarily intended to screen for wide binary companions in anticipation of future observations with non-redundant aperture masking.

Finally, the last six targets we consider (DH Tau, GQ Lup, 1RXSJ1609, UScoJ1610-1935, GSC 6214-210, and ScoPMS 214) were identified as confirmed or likely low-mass companions to young stars in Taurus (DH Tau; Itoh et al. 2005), Lupus (GQ Lup; Neuhäuser et al. 2005), and Upper Sco (the remaining four; Lafrenière et al. 2008; Kraus \& Hillenbrand 2009b; Metchev \& Hillenbrand 2009; Ireland et al. 2011). All were shown to be comoving with their neighbor star and hence are generally assumed to be associated. The only exception is the companion to ScoPMS 214, which Metchev \& Hillenbrand (2009) show to have a spectral type inconsistent with the expected luminosity for a faint companion; the implication is that it is likely to be an unassociated field star with similar motion to Upper Sco. We will revisit this identification in Section 4.3.

\subsection{Past Observations}

Many of the candidate companions in our sample were initially (or also) identified by other observing programs, as were several confirmed PMCs that we are also studying. We have collated all observations for these targets in Table 2 and will combine them with our own measurements in order to test the association of candidate companions and measure a uniform set of colors for confirmed PMCs.

We have adopted most of these observations as stated in their sources, but several seem to require updated parameters. The observation of PDS 70 by Riaud et al. (2006) did not include a position angle (P.A.), so we have measured its P.A. from images downloaded from the European Southern Observatory archive. The observation of FW Tau by White \& Ghez (2001) included a P.A. that is discrepant by $\sim 111^{\circ}$ from our own measurements, so we also measured an updated value of the P.A. using the processed images from the Hubble Legacy Archive. In both cases, we conservatively assess an uncertainty of $\sim 0.5$, corresponding to the angle subtended by the FWHM of the companion 
Host Stars for Candidate or Confirmed PMCs

\begin{tabular}{|c|c|c|c|c|c|c|c|c|c|c|c|c|c|c|c|c|}
\hline Name & 2MASS $J$ & $m_{r^{\prime}}$ & $m_{J}$ & $\begin{array}{c}m_{H} \\
(\mathrm{ma}\end{array}$ & $m_{K s}$ & $m_{W 1}$ & $m_{W 3}$ & $\begin{array}{c}\mu \\
\left(\operatorname{mas~yr}^{-1}\right)\end{array}$ & $\mathrm{SpT}$ & $\begin{array}{c}A_{V} \\
\text { (mag) }\end{array}$ & $\begin{array}{l}\text { Dist } \\
(\mathrm{pc})\end{array}$ & $\begin{array}{l}T_{\text {eff }} \\
(\mathrm{K})\end{array}$ & $\begin{array}{c}M_{\text {bol }} \\
(\mathrm{mag})\end{array}$ & $\begin{array}{l}\log (\tau) \\
((\mathrm{Myr})\end{array}$ & $\begin{array}{c}M \\
\left(M_{\odot}\right)\end{array}$ & Refs \\
\hline \multicolumn{17}{|c|}{ New Companion Hosts } \\
\hline FW Tau ${ }^{\mathrm{a}}$ & $04292971+2616532$ & 15.31 & 10.34 & 9.68 & 9.39 & 9.19 & 8.79 & $(8.9,-28.1) \pm 3.3$ & M4 & 2.1 & 145 & $3270_{-70}^{+70}$ & $6.52 \pm 0.26$ & $6.26 \pm 0.18$ & $0.28 \pm 0.05$ & 1 \\
\hline ROXs 12 & $16262774-2527247$ & 15.80 & 11.02 & 9.93 & 9.21 & 8.43 & 5.99 & $(-9.5,-30.0) \pm 3.4$ & M0 & 4.5 & 120 & $3850_{-70}^{+100}$ & $5.94 \pm 0.26$ & $6.88 \pm 0.19$ & $0.87 \pm 0.08$ & 2 \\
\hline ROXs $42 \mathrm{~B}^{\mathrm{a}}$ & $16311501-2432436$ & 13.58 & 9.91 & 9.02 & 8.67 & 8.45 & 8.23 & $(-8.8,-14.6) \pm 3.0$ & M0 & 2.4 & 120 & $3850_{-70}^{+100}$ & $5.74 \pm 0.26$ & $6.83 \pm 0.18$ & $0.89 \pm 0.08$ & 2 \\
\hline \multicolumn{17}{|c|}{ Known Companion Hosts } \\
\hline$\overline{\mathrm{DH}}$ Tau & $04294155+2632582$ & 13.16 & 9.77 & 8.82 & 8.18 & 7.11 & 5.69 & $(7.1,-17.9) \pm 3.4$ & M1 & 1.4 & 145 & $3705_{-70}^{+70}$ & $5.21 \pm 0.26$ & $6.21 \pm 0.17$ & $0.82 \pm 0.09$ & 3 \\
\hline GQ Lup & 15491210-3539051 & 11.22 & 8.61 & 7.70 & 7.10 & 6.07 & 4.34 & $(-15.1,-23.4) \pm 2.7$ & K7 & 1.5 & 155 & $4060_{-100}^{+150}$ & $3.67 \pm 0.26$ & $6.09 \pm 0.12$ & $1.37 \pm 0.10$ & 4 \\
\hline 1RXS J1609 & 16093030-2104589 & 12.11 & 9.82 & 9.12 & 8.92 & 8.77 & 8.72 & $(-11.2,-21.9) \pm 1.5$ & K7 & 0.9 & 145 & $4060_{-100}^{+150}$ & $5.19 \pm 0.27$ & $6.79 \pm 0.21$ & $1.08 \pm 0.08$ & 5 \\
\hline UScoJ1610-1913 & 16103196-1913062 & 12.84 & 10.03 & 9.26 & 8.99 & 8.70 & 8.53 & $(-9.8,-22.5) \pm 1.9$ & K7 & 1.9 & 145 & $4060_{-100}^{+150}$ & $5.12 \pm 0.27$ & $6.76 \pm 0.20$ & $1.09 \pm 0.08$ & 6 \\
\hline GSC $6214-210$ & $16215466-2043091$ & 11.94 & 10.00 & 9.34 & 9.15 & 9.08 & 8.96 & $(-18.6,-32.2) \pm 1.7$ & K7 & 0.2 & 145 & $4060_{-100}^{+150}$ & $5.57 \pm 0.27$ & $6.99 \pm 0.20$ & $1.01 \pm 0.07$ & 7 \\
\hline \multicolumn{17}{|c|}{ Neighbors of Background Stars } \\
\hline $2 \mathrm{M} 0415+2818$ & $04153916+2818586$ & 15.34 & 10.55 & 9.61 & 9.24 & 8.75 & 6.89 & $(11.6,-26.0) \pm 3.6$ & M3.75 & 2.0 & 130 & $3305_{-70}^{+70}$ & $6.24 \pm 0.26$ & $6.17 \pm 0.15$ & $0.34 \pm 0.06$ & 8 \\
\hline HD $27659^{\mathrm{b}}$ & $04225462+2823540$ & 8.28 & $\ldots$ & $\ldots$ & 7.25 & 7.33 & 6.78 & $(-21.3,-23.3) \pm 7.6$ & A4 & 1.1 & 130 & $8500_{-1000}^{+1000}$ & $1.52 \pm 0.26$ & $6.96 \pm 0.20$ & $2.14 \pm 0.20$ & 8 \\
\hline PDS 70 & $14081015-4123525$ & 11.15 & 9.55 & 8.82 & 8.54 & 8.03 & 5.72 & $(-32.4,-28.5) \pm 1.7$ & K5 & 0.0 & 140 & $4350_{-150}^{+120}$ & $5.30 \pm 0.26$ & $7.08 \pm 0.20$ & $1.15 \pm 0.09$ & 9 \\
\hline GSC 06191-00019a & $15590208-1844142$ & 11.51 & 9.00 & 8.34 & 8.11 & 7.93 & 7.75 & $(-8.5,-24.8) \pm 1.9$ & K6 & 1.4 & 145 & $4350_{-150}^{+120}$ & $4.70 \pm 0.26$ & $6.78 \pm 0.21$ & $1.32 \pm 0.10$ & 7 \\
\hline UScoJ1608-1935 & 16082387-1935518 & 13.57 & 10.20 & 9.47 & 9.25 & 9.07 & 8.91 & $(-10.5,-25.7) \pm 2.3$ & M1 & 1.4 & 145 & $3705_{-70}^{+70}$ & $5.65 \pm 0.26$ & $6.57 \pm 0.18$ & $0.75 \pm 0.08$ & 7 \\
\hline ScoPMS 42b & $16102174-1904067$ & 14.23 & 10.68 & 9.91 & 9.62 & 9.31 & 9.09 & $(-12.5,-25.8) \pm 3.1$ & M1 & 1.7 & 145 & $3705_{-70}^{+70}$ & $6.04 \pm 0.27$ & $6.76 \pm 0.19$ & $0.72 \pm 0.08$ & 7 \\
\hline GSC 06793-00994 & $16140211-2301021$ & 11.07 & 9.38 & 8.77 & 8.61 & 8.41 & 8.35 & $(-7.1,-20.7) \pm 0.8$ & G4 & 1.3 & 145 & $5840_{-35}^{+35}$ & $4.23 \pm 0.27$ & $7.72 \pm 0.19$ & $1.20 \pm 0.04$ & 7 \\
\hline GSC 06794-00156 & $16245136-2239325$ & 9.40 & 7.78 & 7.28 & 7.08 & 6.99 & 7.00 & $(-11.8,-22.1) \pm 1.4$ & G6 & 1.0 & 145 & $5700_{-35}^{+35}$ & $3.27 \pm 0.27$ & $7.24 \pm 0.07$ & $1.35 \pm 0.04$ & 7 \\
\hline DoAr 22 & $16261932-2343205$ & 12.18 & 9.73 & 9.25 & 9.02 & 8.82 & 8.62 & $(4.5,-20.0) \pm 1.3$ & F5 & 3.1 & 120 & $6440_{-400}^{+800}$ & $4.23 \pm 0.26$ & $7.89 \pm 0.34$ & $1.24 \pm 0.05$ & 2 \\
\hline ScoPMS 214 & $16294869-2152118$ & 10.92 & 8.68 & 8.00 & 7.76 & 7.61 & 7.52 & $(-9.9,-23.9) \pm 1.2$ & K0 & 1.9 & 145 & $5250_{-170}^{+170}$ & $3.55 \pm 0.27$ & $7.02 \pm 0.19$ & $1.40 \pm 0.05$ & 10 \\
\hline
\end{tabular}

Notes.

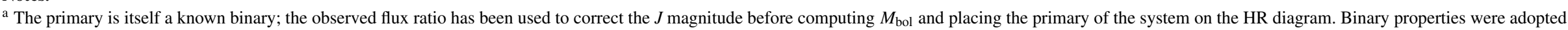
from Kraus et al. (2011) for FW Tau, Ratzka et al. (2005) for ROXs 42B, and Kraus et al. (2008) for UScoJ1610-1913, GSC 06191-00019, and GSC 06794-00156.

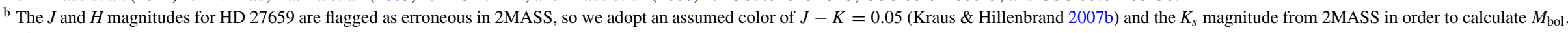

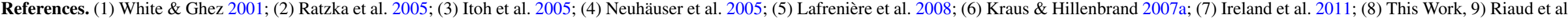
2006; (10) Metchev \& Hillenbrand 2009. 
Table 2

Literature Measurements of Known and Candidate PMCs

\begin{tabular}{|c|c|c|c|c|c|c|}
\hline Name & $\begin{array}{c}\text { JD } \\
(-2400000)\end{array}$ & Filter & $\begin{array}{c}\rho \\
\text { (mas) }\end{array}$ & $\begin{array}{l}\text { P.A. } \\
\text { (deg) }\end{array}$ & $\begin{array}{c}\Delta m \\
(\mathrm{mag})\end{array}$ & Ref \\
\hline 1RXS J1609 & 54584 & $K_{s}$ & $2215 \pm 6$ & $27.75 \pm 0.10$ & $7.25 \pm 0.18$ & 1 \\
\hline 1RXS J1609 & 54635 & $K^{\prime}$ & $2210 \pm 2$ & $27.62 \pm 0.05$ & $7.27 \pm 0.05$ & 2 \\
\hline 1RXS J1609 & 54639 & $H$ & $2222 \pm 6$ & $27.76 \pm 0.10$ & $7.75 \pm 0.07$ & 1 \\
\hline 1RXS J1609 & 54639 & $J$ & $2219 \pm 6$ & $27.76 \pm 0.10$ & $8.08 \pm 0.12$ & 1 \\
\hline 1RXS J1609 & 54928 & $K^{\prime}$ & $2222 \pm 6$ & $27.65 \pm 0.10$ & $\ldots$ & 1 \\
\hline 1RXS J1609 & 54983 & $K^{\prime}$ & $2211 \pm 2$ & $27.61 \pm 0.05$ & $7.23 \pm 0.05$ & 2 \\
\hline 1RXS J1609a & 55014 & $K^{\prime}$ & $2219 \pm 6$ & $27.74 \pm 0.10$ & & 1 \\
\hline $1 \mathrm{RXS} \mathrm{J1609}{ }^{\mathrm{b}}$ & 55021 & $L^{\prime}$ & $\ldots$ & $\ldots$ & $6.1 \pm 0.3$ & 1 \\
\hline DH Tau ${ }^{\mathrm{a}}$ & 51196 & $R_{C} I_{C}$ & $2351 \pm 2$ & $139.36 \pm 0.10$ & $\ldots$ & 3 \\
\hline DH Tau ${ }^{\mathrm{a}}$ & 52602 & $H$ & $2340 \pm 6$ & $139.56 \pm 0.17$ & $\ldots$ & 3 \\
\hline DH Tau & 53013 & $K$ & $2344 \pm 3$ & $139.83 \pm 0.06$ & $6.01 \pm 0.05$ & 3 \\
\hline DH Tau ${ }^{\mathrm{b}}$ & 53013 & $H$ & $\ldots$ & $\ldots$ & $6.14 \pm 0.05$ & 3 \\
\hline DH Tau ${ }^{\mathrm{b}}$ & 53013 & $J$ & $\ldots$ & $\ldots$ & $5.94 \pm 0.05$ & 3 \\
\hline DoAr 22 & 52093 & $K$ & $2297 \pm 30$ & $258.9 \pm 0.7$ & $5.75 \pm 0.11$ & 4 \\
\hline $\mathrm{FW} \mathrm{Tau}{ }^{\mathrm{a}}$ & 50526 & $R_{C} I_{C} H \alpha$ & $2295 \pm 3$ & $295.0 \pm 0.5$ & $\ldots$ & 5 \\
\hline GQ Lup & 49445 & K & $714 \pm 36$ & $275.5 \pm 1.1$ & $6.24 \pm 0.13$ & 6 \\
\hline GQ Lup ${ }^{a}$ & 51279 & $R_{C} I_{C}$ & $739 \pm 11$ & $275.62 \pm 0.86$ & $\ldots$ & 7 \\
\hline GQ Lup ${ }^{b}$ & 52473 & $K$ & $\ldots$ & $\ldots$ & $6.27 \pm 0.12$ & 8 \\
\hline GQ Lup ${ }^{b}$ & 52473 & $L^{\prime}$ & $\ldots$ & $\ldots$ & $6.39 \pm 0.22$ & 8 \\
\hline GQ Lup ${ }^{\mathrm{b}}$ & 52473 & $\mathrm{CH} 4 \mathrm{~S}$ & $\ldots$ & $\ldots$ & $6.06 \pm 0.26$ & 8 \\
\hline GQ Lup ${ }^{a}$ & 53182 & $K_{s}$ & $734.7 \pm 3$ & $275.48 \pm 0.25$ & $\ldots$ & 9 \\
\hline GQ Lup ${ }^{b}$ & 53182 & $K_{s}$ & $\ldots$ & $\ldots$ & $6.00 \pm 0.10$ & 7 \\
\hline GQ Lup & 53518 & $K_{s}$ & $735.1 \pm 3$ & $276.00 \pm 0.34$ & $6.39 \pm 0.12$ & 9 \\
\hline GQ Lup & 53591 & $K_{s}$ & $733.3 \pm 4$ & $275.87 \pm 0.37$ & $6.3 \pm 0.14$ & 9 \\
\hline GQ Lup & 53789 & $K_{s}$ & $729.8 \pm 3$ & $276.14 \pm 0.35$ & $6.29 \pm 0.10$ & 9 \\
\hline GQ Lup & 53876 & $K_{s}$ & $731.4 \pm 4$ & $276.06 \pm 0.38$ & $6.07 \pm 0.14$ & 9 \\
\hline GQ Lup & 53933 & $K_{s}$ & $733.2 \pm 5$ & $276.26 \pm 0.68$ & $6.43 \pm 0.38$ & 9 \\
\hline GQ Lup & 54151 & $K_{s}$ & $730.0 \pm 6$ & $276.04 \pm 0.63$ & $5.79 \pm 0.12$ & 9 \\
\hline GSC 06214-00210 & 54258 & $K^{\prime}$ & $2203 \pm 2$ & $176.04 \pm 0.06$ & $5.74 \pm 0.05$ & 2 \\
\hline GSC 06214-00210 & 54635 & $K^{\prime}$ & $2205 \pm 2$ & $175.99 \pm 0.05$ & $5.78 \pm 0.05$ & 2 \\
\hline GSC 06214-00210 & 54635 & $J$ & $2205 \pm 2$ & $176.00 \pm 0.09$ & $6.3 \pm 0.05$ & 2 \\
\hline GSC 06214-00210 & 54983 & $K^{\prime}$ & $2204 \pm 2$ & $175.91 \pm 0.05$ & $5.88 \pm 0.05$ & 2 \\
\hline GSC 06214-00210 & 55313 & $K^{\prime}$ & $2206 \pm 2$ & $175.93 \pm 0.05$ & $5.73 \pm 0.05$ & 2 \\
\hline GSC 06214-00210 & 55313 & $H$ & $2203 \pm 2$ & $175.91 \pm 0.05$ & $6.21 \pm 0.05$ & 2 \\
\hline GSC 06214-00210 & 55313 & $L^{\prime}$ & $\ldots$ & $\ldots$ & $4.75 \pm 0.05$ & 2 \\
\hline GSC 06793-00994 & 52836 & $K_{s}$ & $5366 \pm 30$ & $356.1 \pm 0.5$ & $7.76 \pm 0.12$ & 10 \\
\hline GSC 06793-00994 & 54251 & $K_{s}$ & $5462 \pm 17$ & $357.4 \pm 0.2$ & $7.79 \pm 0.05$ & 2 \\
\hline GSC 06794-00156 & 54251 & $K_{s}$ & $5973 \pm 18$ & $338.7 \pm 0.2$ & $9.43 \pm 0.05$ & 2 \\
\hline PDS 70 & 53574 & $K$ & $2155 \pm 2$ & $6.3 \pm 0.5$ & $4.68 \pm 0.05$ & 11 \\
\hline ROXs 12 & 52093 & $K$ & $1747 \pm 30$ & $10.3 \pm 0.9$ & $5.75 \pm 0.11$ & 4 \\
\hline ROXs 42B & 52092 & $K$ & $1137 \pm 30$ & $268.0 \pm 1.5$ & $6.75 \pm 0.40$ & 4 \\
\hline ScoPMS 214 & 52517 & $K s$ & $3070 \pm 10$ & $121.17 \pm 0.23$ & $5.96 \pm 0.09$ & 10 \\
\hline UScoJ1610-1913 ${ }^{\mathrm{a}}$ & 51297 & $H$ & $5872 \pm 70$ & $112.6 \pm 0.7$ & $\ldots$ & 13 \\
\hline UScoJ1610-1913 ${ }^{\mathrm{a}}$ & 51297 & $J$ & $5762 \pm 70$ & $114.1 \pm 0.7$ & $\ldots$ & 13 \\
\hline UScoJ1610-1913 ${ }^{\mathrm{a}}$ & 51297 & K & $5965 \pm 70$ & $113.6 \pm 0.7$ & $\ldots$ & 13 \\
\hline UScoJ1610-1913 & 54253 & $K s$ & $5820 \pm 9$ & $114.01 \pm 0.10$ & $3.83 \pm 0.05$ & 14 \\
\hline
\end{tabular}

Notes.

${ }^{a}$ This observation is only for astrometry; the photometric measurement was too uncertain to be reliable, was reported elsewhere, or was not reported.

$\mathrm{b}$ This observation is only for photometry; the astrometric measurement was too uncertain to be reliable, was reported elsewhere, or was not reported. References. (1) Lafrenière et al. 2010; (2) Ireland et al. 2011; (3) Itoh et al. 2005; (4) Ratzka et al. 2005; (5) White \& Ghez 2001; (6) Janson et al. 2006; (7) Neuhäuser et al. 2005; (8) Marois et al. 2007; (9) Neuhäuser et al. 2008; (10) Metchev \& Hillenbrand 2009; (11) Riaud et al. 2006; (12) Köhler et al. 2000; (13) Kraus \& Hillenbrand 2009b; (14) Kraus et al. 2008.

point-spread function (PSF). Finally, the observations reported by Ratzka et al. (2005) for several companions (including two comoving companions) disagree with other observations by significantly more than their reported uncertainties (which are typically $<10$ mas). Since they reported the detections at signalto-noise ratios $\sim 5$ from shift-and-add speckle imaging, then we expect that the minimum positional uncertainties should be $\sim 1 / 5 \lambda / D$ or $\sim 30$ mas. We therefore have adopted uncertainties of 30 mas for their projected separations and the corresponding subtended angle for their P.A.s.

More generally, we note that many observations seem to base their uncertainties on the scatter in their measurements, without considering systematic errors. We therefore also have adopted the systematic uncertainty floors that we assign to our own NIRC2 data (which are typically more stable than data from most other instruments). Due to uncorrected residuals 
from geometric distortion (e.g., Anderson \& King 2003; Yelda et al. 2010), all astrometry is given a minimum uncertainty of 2 mas. Furthermore, due to the uncertain plate scale, all projected separations are given a minimum fractional uncertainty of $10^{-3}$. Finally, due to anisoplanatism and variability of the host stars, all contrast ratios are given a minimum uncertainty of $0.05 \mathrm{mag}$. These uncertainties could be larger for targets observed using multiple telescopes, instruments, and observing techniques. However, there has never been a global calibration of the plate scale of geometric distortion (such as with observations of globular cluster fields) for any significant fraction of high-resolution imaging instruments.

\subsection{Properties of the Host Stars}

We obtained photometric data for the host stars of each candidate from publicly available all-sky surveys. The optical $r^{\prime}$ magnitudes for most host stars were taken from the 14th Carlsberg Meridianal Catalog (CMC14; Evans et al. 2002). Some targets are too far south or otherwise do not have CMC14 counterparts, so for those stars we adopted the average of the USNO B1.0 $R$ magnitudes (Monet et al. 2003). Based on the stars that have measurements in both catalogs, the magnitudes are equivalent to within the uncertainties $\left(R_{\mathrm{USNOB}}-r^{\prime} \sim 0\right)$. The $1.0-2.5 \mu \mathrm{m}$ $\left(J, H\right.$, and $\left.K_{s}\right)$ data were obtained from 2MASS (Skrutskie et al. 2006). Most of our AO imaging measurements were taken with a $K^{\prime}$ filter $(\lambda=2.124 \mu \mathrm{m})$, while measurements from the literature often use $K(\lambda=2.196 \mu \mathrm{m})$ or $K_{s}(\lambda=2.146 \mu \mathrm{m})$, but the conversion terms for late-M and early-L objects are negligible ( $\lesssim 0.01-0.02 \mathrm{mag}$; Carpenter 2001) compared with the typical systematic uncertainties of AO photometry. Finally, the $3.0-15 \mu \mathrm{m}$ data ( $W 1$ and $W 3$ ) were obtained from WISE (Wright et al. 2010). The WISE W1 filter is centered at $3.35 \mu \mathrm{m}(2.9-3.8 \mu \mathrm{m})$, while our ground-based measurements were taken with a Mauna Kea Observatories (MKO) $L^{\prime}$ filter centered at $3.78 \mu \mathrm{m}(3.4-4.1 \mu \mathrm{m})$, so use of WISE data requires a color correction. The conversion has not yet been reported in the literature, so we downloaded WISE data for 40 M3.0-L9.5 field dwarfs that have $L^{\prime}$ data reported in the literature (Leggett et al. 2010) and computed a color-SpT relation of $W 1-L^{\prime}=$ $0.044 \times \operatorname{SpT}-0.078$ (where SpT $=0$ for M0 and 20 for T0), which has a scatter about the relation of $\sigma=0.10 \mathrm{mag}$ for the full sample and $\sigma=0.05$ mag specifically for M5-L1 dwarfs. We tested this relation for the isolated BDs observed with $L^{\prime}$ photometry by Jayawardhana et al. (2003) and found that the mean $W 1-L^{\prime}$ colors agreed to within $0.2 \mathrm{mag}$, with a scatter of $0.2 \mathrm{mag}$; similar offsets were seen for disk-bearing and disk-free BDs, indicating that infrared excesses do not bias this relation.

None of the host stars were observed by Hipparcos or otherwise have direct trigonometric parallaxes, so we must infer their distances from the mean and standard deviation of other members of their associations. Based on previous trigonometric parallaxes, we have adopted these values collectively for all members of Taurus (145 \pm 15 pc; Torres et al. 2009), Ophiuchus $(120 \pm 10$ pc; Loinard et al. 2008), Upper Sco (145 \pm 15 pc; de Zeeuw et al. 1999), and Upper Centaurus-Lupus (140 \pm 15 pc; de Zeeuw et al. 1999). The number of Hipparcos parallaxes toward the Lupus dark clouds is small, so we instead adopt the extinction-based measurement of $155 \pm 15$ pc reported by Lombardi et al. (2008), which is consistent with the convergentpoint distance recently reported by Galli et al. (2013). Where available, we adopt proper motions for each star that were reported in the UCAC3 catalog (Zacharias 2010). However, some targets are too optically faint for UCAC3, so we instead report proper motions derived from USNO-B1, 2MASS, and Sloan Digital Sky Survey (SDSS) using the methods described in Kraus \& Hillenbrand (2007b).

We have adopted the spectral types for the host stars as reported in the literature. Many of the host stars also have extinction measurements reported in the literature. However, some are missing such measurements and others appear to be significantly redder than expected for the given spectral type and $A_{V}$. We therefore have calculated new extinction estimates by comparing each star's observed $r^{\prime}-J$ or $R-J$ color to that for field dwarfs of the same spectral type (Kraus \& Hillenbrand $2007 b$ ), converting the color excess to a visual extinction using the extinction relations of Schlegel et al. (1998). Most of our new measurements agree to within $\Delta A_{V} \lesssim 1$ or $\Delta A_{J} \lesssim 0.25$. However, two stars (ROXs 12 and DoAr 22) are significantly more extincted than reported in the literature $\left(\Delta A_{V} \sim 3\right)$.

We estimated the temperatures of the host stars based on their spectral types, combined with the dwarf temperature scale of Schmidt-Kaler (1982) for $\leqslant$ M0 stars and the pre-main sequence temperature scale of Luhman et al. (2003) for $>$ M0 stars. We estimated their luminosities from their dereddened 2MASS $J$ magnitudes, the inferred distances listed above, and the $J$ band bolometric corrections we previously tabulated in Kraus \& Hillenbrand (2007b). Finally, we used these temperatures and luminosities to place each host star on the Hertzsprung-Russell (HR) diagram, where they can be directly compared with the predictions of pre-main sequence evolutionary models. Different models make highly discrepant predictions, but based on past calibrations (as summarized by Hillenbrand \& White 2004) and the dynamical masses that we have measured in these regions (e.g., M. J. Ireland et al., in preparation; Kraus et al. 2014), we have chosen the models of Baraffe et al. (1998) using a convective mixing length of $1 H_{P}$. The star HD 27659 falls outside the temperature range spanned by those models, so we instead used the models of Siess et al. (2000). This said, its proper motion is not consistent with Taurus membership, so an age and mass derived for the distance of Taurus might not be meaningful.

We summarize the properties of these host stars in Table 1.

\section{NEW OBSERVATIONS AND CANDIDATE PROPERTIES}

\subsection{High-resolution Imaging Observations and Data Analysis}

Most of our high-resolution imaging observations were obtained at Keck Observatory using the Keck-II 10m telescope and NIRC2, its facility AO imager. GSC 06191-00019 was also observed at Palomar Observatory using the Palomar-Hale 200" telescope and PHARO, its facility AO imager (Hayward et al. 2001). All of the primary stars are brighter than $R=15$, so we observed them with natural guide star AO at both Keck and Palomar.

The observations span a number of observing seasons (2007-2013) and were taken by several different observers, so they vary significantly in their details (such as total integration time, dither pattern, and Fowler sampling). Several observations using NIRC2 in $K^{\prime}$ were also taken using the 600 mas coronagraphic spot, which provides $\Delta K^{\prime}=7.17 \pm 0.10$ mag of attenuation for the primary star (as measured from observations of binary stars). All observations used the smallest pixel scales (10 mas/pix with NIRC2 and 25 mas/pix with PHARO). We summarize the salient details for these observations in Table 3 
and also refer readers to the Keck Observatory Archive, ${ }^{9}$ which now hosts all NIRC2 data after an 18-month proprietary period.

The data analysis follows the same prescription as described in Kraus et al. (2008). To briefly summarize, we measured astrometry and aperture photometry for each source with respect to the purported primary star using the IRAF task DAOPHOT (Stetson 1987); all measurements were conducted using apertures of $0.5,1.0$, and $2.0 \lambda / D$ and then the optimal aperture was chosen to maximize the significance of the detection (given the competing uncertainties from the primary star's PSF halo and the Poisson noise for the candidate companion). In order to estimate the uncertainties from the data, we analyzed the measurements in individual frames and then combined those measurements to estimate the mean and standard deviation. We then corrected for geometric distortion in the images, accounting for the remaining systematic uncertainty in the plate scales and distortion solutions of PHARO (0.3\%; Ireland et al. 2011) and NIRC2 (0.05\%; Ghez et al. 2008; Cameron 2008) by adding those terms in quadrature with the observed scatter. Candidates were judged to be comoving if their relative proper motion fell within $<3 \sigma$ of zero and disagreed with the expected motion of a background star by $>3 \sigma$.

In Table 3, we list the observations and results for all candidate companions that we observed.

\subsection{Candidate Properties}

Young stars are intrinsically variable due to several phenomena (i.e., spots, accretion, and variable extinction), so the conversion of contrasts into magnitudes and colors is subject to systematic uncertainties. Furthermore, the precision of $\mathrm{AO}$ photometry is fundamentally limited by anisoplanatism (e.g., Steinbring et al. 2002), especially for relatively faint targets that were observed with modest Strehl ratios. To average down these uncertainties, we have combined all available photometric measurements for each object to compute a weighted mean contrast in each filter, with an additional caveat that no epoch was given an uncertainty better than $\sim 0.05$ mag. We then computed apparent magnitudes by adding each contrast measurement to the known magnitude for its parent star (Section 2.3; Table 1), adding the uncertainties in quadrature and also assessing a systematic uncertainty of $\sim 0.05$ mag in the brightness of the primary (due to its potential variability at the 2MASS epoch; Carpenter et al. 2002). We report the resulting apparent magnitudes in Table 4.

We measured the relative motion of each object with a weighted linear fit to compute $\left(\mu_{r e l, \alpha}, \mu_{r e l, \delta}\right)$. Due to the precise and accurate calibration of NIRC2 astrometry (e.g., Yelda et al. 2010), then any fit with multiple NIRC2 points (including those of the three bona fide companions) is effectively dominated by the NIRC2 measurements. If the companion was not associated, then there also would be an error term for parallactic motion, but such motions are small compared with the proper motion over timescales of $>1 \mathrm{yr}$. We report the relative motions and the inferred physical projected separations in Table 4.

For the bona fide companions, the absolute magnitudes of the companions were converted into masses (in $M_{\mathrm{Jup}}$ ) using the hot-start DUSTY models, which are more appropriate than the COND models due to the dusty nature of young low-temperature photospheres (Cruz et al. 2009; Allers et al. 2010; Bowler et al. 2010; Patience et al. 2010). The reported uncertainties are dominated by the primary stars' poorly constrained ages, which leave

\footnotetext{
9 http://www2.keck.hawaii.edu/koa/public/koa.php
}

the masses uncertain by up to $50 \%$; the photometric uncertainties are negligible in comparison. The plausibility of the models adds another significant systematic uncertainty; newer models suggest that the hot-start models could significantly underestimate planet masses (Fortney et al. 2008; Spiegel \& Burrows 2012; Marleau \& Cumming 2013).

In each case, we use the hot-start DUSTY models to estimate a range of plausible masses based on the age range seen in its host region (1-5 Myr for Taurus, Ophiuchus, and Lupus; 5-10 Myr for Upper Sco), and assign a best-fit mass that is the average of these minimum and maximum limits. These age ranges encompass a possible upward revision of all premain sequence stellar ages (e.g., Pecaut et al. 2012) and hence allow for higher masses than were previously estimated. We will discuss our estimates for the companion masses in more detail in Section 4 and list the mass ranges in Table 4.

\section{RESULTS}

\subsection{Three PMCs to Young Stars}

As we summarized in Table 4, three of the candidate companions in our sample are comoving with their host stars. Each is located in a wide orbit ( $\rho \gtrsim 150 \mathrm{AU})$ and has an apparently planetary mass $\left(M=6-20 M_{\text {Jup }}\right)$. All were previously reported in the literature as candidate binary companions, but have been neglected for the past decade. Based on the two-point correlation function in unbound associations like these regions (Kraus \& Hillenbrand 2008), the probability of chance alignment with an unbound association member within $<3^{\prime \prime}$ is small $(<1$ chance alignment, including stellar-mass companions, per $10^{4}$ members) and hence these objects represent a population of bound companions near or within the planetary-mass regime.

\subsubsection{FW Tau b}

FW Tau AB is located near the center of the Taurus-Auriga complex and is a close ( $\rho \sim 75$ mas; 11 AU) binary system comprised of two stars with near-equal fluxes at wavelengths of $0.3-2.2 \mu \mathrm{m}$ and equal fluxes in the $K^{\prime}$ band (White $\&$ Ghez 2001). Given the unresolved system spectral type of M4 (Briceno et al. 1993), models suggest individual masses of $0.28 \pm 0.05 M_{\odot}$ for each component. The corresponding isochronal age is $1.8_{-0.5}^{+1.0} \mathrm{Myr}$, consistent with the median age of Taurus-Auriga (1.8 Myr; Kraus \& Hillenbrand 2009a). Observations of the $H \alpha$ emission line strength $(\mathrm{EW}[\mathrm{H} \alpha]=-17 \AA$; Briceno et al. 1993) and the lack of excess emission from nearinfrared (NIR) and mid-infrared photometry (Rebull et al. 2010) suggest that the primary is not accreting and does not host a substantial disk around either component or within the inner $\sim 50$ AU. However, Andrews \& Williams (2005) reported a $4 \sigma$ detection of submm flux $\left(F_{v}=4.5 \pm 1.1 \mathrm{mJy}\right)$ that could indicate a disk with a very low mass $\left(M_{\text {disk }} \sim 0.2 M_{\text {Jup }}\right)$ located around one or more objects in the system.

The candidate wide companion to FW Tau was first identified by White \& Ghez (2001), who noted a faint object at a projected separation of $\sim 2$ ". $3(\sim 330$ AU) from the primary star of the central binary. This candidate companion was marginally detected by $H S T$ imaging in the F675W $\left(R_{C}\right)$ and F814W $\left(I_{C}\right)$ filters, as well as having a significant detection in the narrowband F656N $(H \alpha)$ filter. They concluded from its apparently significant $H \alpha$ line emission that it could be an accreting companion. However, no further observations of the candidate companion have since been reported. 
Table 3

New Observations of Known and Candidate PMCs

\begin{tabular}{|c|c|c|c|c|c|c|c|}
\hline Name & $\begin{array}{c}\mathrm{JD} \\
(-2400000)\end{array}$ & Filter & $\begin{array}{l}t_{\text {int }} \\
(\mathrm{sec})\end{array}$ & $\begin{array}{c}\rho \\
\text { (mas) }\end{array}$ & $\begin{array}{l}\text { P.A. } \\
(\mathrm{deg})\end{array}$ & $\begin{array}{c}\Delta m \\
(\mathrm{mag})\end{array}$ & $\mathrm{Tel} / \mathrm{Inst}$ \\
\hline 1RXS J1609 & 55736 & $K^{\prime}$ & 80 & $2211 \pm 2$ & $27.61 \pm 0.08$ & $7.43 \pm 0.04$ & Keck-NIRC2 \\
\hline $2 \mathrm{M} 0415+2818$ & 55157 & $K^{\prime}$ & 50 & $2620 \pm 3$ & $89.13 \pm 0.04$ & $5.67 \pm 0.02$ & Keck-NIRC2 \\
\hline DH Tau & 54824 & $J$ & 60 & $2354 \pm 2$ & $139.38 \pm 0.05$ & $5.90 \pm 0.02$ & Keck-NIRC2 \\
\hline DH Tau & 54824 & $K^{\prime}$ & 40 & $2352 \pm 2$ & $139.35 \pm 0.05$ & $5.92 \pm 0.02$ & Keck-NIRC2 \\
\hline DH Tau & 54824 & $H$ & 40 & $2354 \pm 2$ & $139.39 \pm 0.05$ & $5.97 \pm 0.05$ & Keck-NIRC2 \\
\hline DH Tau & 56310 & $L^{\prime}$ & 160 & $2350 \pm 2$ & $139.39 \pm 0.05$ & $5.82 \pm 0.02$ & Keck-NIRC2 \\
\hline DoAr 22 & 56022 & $K^{\prime}$ & 70 & $2480 \pm 2$ & $265.32 \pm 0.05$ & $5.97 \pm 0.02$ & Keck-NIRC2 \\
\hline DoAr 22 & 55735 & $K^{\prime}$ & 110 & $2469 \pm 2$ & $264.92 \pm 0.05$ & $5.94 \pm 0.01$ & Keck-NIRC2 \\
\hline DoAr 22 & 55735 & $J$ & 36 & $2468 \pm 2$ & $264.93 \pm 0.05$ & $6.22 \pm 0.01$ & Keck-NIRC2 \\
\hline FW Tau & 54824 & $K^{\prime}$ & 40 & $2282 \pm 2$ & $295.53 \pm 0.08$ & $5.91 \pm 0.03$ & Keck-NIRC2 \\
\hline FW Tau & 54824 & $H$ & 80 & $2285 \pm 2$ & $295.48 \pm 0.08$ & $6.57 \pm 0.02$ & Keck-NIRC2 \\
\hline FW Tau & 54824 & $J$ & 120 & $2280 \pm 2$ & $295.34 \pm 0.17$ & $7.00 \pm 0.04$ & Keck-NIRC2 \\
\hline FW Tau & 55849 & $K^{\prime}$ & 100 & $2278 \pm 2$ & $295.56 \pm 0.05$ & $5.94 \pm 0.03$ & Keck-NIRC2 \\
\hline FW Tau & 56152 & $L^{\prime}$ & 140 & $2273 \pm 5$ & $295.50 \pm 0.07$ & $5.15 \pm 0.06$ & Keck-NIRC2 \\
\hline GQ Lup & 56023 & $K^{\prime}$ & 40 & $722.7 \pm 1.2$ & $277.25 \pm 0.16$ & $6.32 \pm 0.01$ & Keck-NIRC2 \\
\hline GQ Lup & 56023 & $H$ & 50 & $717.3 \pm 1.2$ & $277.37 \pm 0.16$ & $6.09 \pm 0.04$ & Keck-NIRC2 \\
\hline GQ Lup & 56023 & $L^{\prime}$ & 30 & $713.0 \pm 2.5$ & $277.61 \pm 0.42$ & $6.21 \pm 0.05$ & Keck-NIRC2 \\
\hline GSC 06191-00019 & 54251 & $K_{s}$ & 57 & $3111 \pm 10$ & $321.73 \pm 0.18$ & $8.41 \pm 0.03$ & Palomar-PHARO \\
\hline GSC 06191-00019 & 55392 & $K^{\prime}$ & 120 & $3203 \pm 3$ & $320.63 \pm 0.06$ & $8.42 \pm 0.07$ & Keck-NIRC2 \\
\hline GSC 06191-00019 & 56054 & $K^{\prime}$ & 91 & $3255 \pm 3$ & $319.93 \pm 0.06$ & $8.49 \pm 0.09$ & Keck-NIRC2 \\
\hline GSC 06214-00210 & 55716 & $K^{\prime}$ & 70 & $2206 \pm 2$ & $175.86 \pm 0.05$ & $5.86 \pm 0.03$ & Keck-NIRC2 \\
\hline GSC 06793-00994 & 56054 & $K^{\prime}$ & 54 & $5537 \pm 6$ & $358.09 \pm 0.02$ & $7.82 \pm 0.05$ & Keck-NIRC2 \\
\hline GSC 06794-00156 & 56054 & $K^{\prime}+$ corona 600 & 40 & $6009 \pm 6$ & $339.38 \pm 0.02$ & $8.91 \pm 0.02$ & Keck-NIRC2 \\
\hline HD 27659 & 55157 & $K_{c}$ & 27 & $1283 \pm 9$ & $27.44 \pm 0.37$ & $6.71 \pm 0.13$ & Keck-NIRC2 \\
\hline HD 27659 & 55849 & $K^{\prime}$ & 500 & $1304.6 \pm 1.3$ & $27.63 \pm 0.09$ & $7.10 \pm 0.10$ & Keck-NIRC2 \\
\hline HD 27659 & 55882 & $K^{\prime}$ & 30 & $1296 \pm 2$ & $27.81 \pm 0.09$ & $6.87 \pm 0.05$ & Keck-NIRC2 \\
\hline HD 27659 & 55882 & $J_{c}$ & 20 & $1294 \pm 5$ & $27.91 \pm 0.09$ & $7.39 \pm 0.11$ & Keck-NIRC2 \\
\hline HD 27659 & 55882 & $K_{c}$ & 90 & $1301.8 \pm 1.6$ & $27.89 \pm 0.09$ & $6.82 \pm 0.08$ & Keck-NIRC2 \\
\hline HD 27659 & 55882 & $H_{c}$ & 131 & $1298.8 \pm 1.3$ & $27.99 \pm 0.09$ & $6.74 \pm 0.01$ & Keck-NIRC2 \\
\hline HD 27659 & 55882 & $J_{c}$ & 90 & $1299.3 \pm 1.3$ & $28.20 \pm 0.09$ & $6.93 \pm 0.02$ & Keck-NIRC2 \\
\hline HD 27659 & 55882 & $L^{\prime}$ & 80 & $1300 \pm 2$ & $28.09 \pm 0.09$ & $6.73 \pm 0.07$ & Keck-NIRC2 \\
\hline PDS 70 & 56022 & $K^{\prime}$ & 60 & $2321 \pm 2$ & $9.98 \pm 0.05$ & $4.64 \pm 0.01$ & Keck-NIRC2 \\
\hline PDS 70 & 56022 & $H$ & 60 & $2321 \pm 2$ & $10.00 \pm 0.05$ & $4.35 \pm 0.03$ & Keck-NIRC2 \\
\hline PDS 70 & 56022 & $J$ & 60 & $2321 \pm 2$ & $10.01 \pm 0.05$ & $4.32 \pm 0.02$ & Keck-NIRC2 \\
\hline PDS 70 & 56022 & $L^{\prime}$ & 90 & $2321 \pm 2$ & $9.95 \pm 0.05$ & $5.27 \pm 0.04$ & Keck-NIRC2 \\
\hline ROXs 12 & 55735 & $K^{\prime}$ & 100 & $1781 \pm 2$ & $9.08 \pm 0.06$ & $5.06 \pm 0.01$ & Keck-NIRC2 \\
\hline ROXs 12 & 55735 & $H$ & 40 & $1780 \pm 2$ & $9.10 \pm 0.06$ & $5.44 \pm 0.01$ & Keck-NIRC2 \\
\hline ROXs 12 & 55735 & $J$ & 50 & $1782 \pm 2$ & $9.08 \pm 0.06$ & $5.62 \pm 0.02$ & Keck-NIRC2 \\
\hline ROXs 12 & 55735 & $L^{\prime}$ & 91 & $1778 \pm 2$ & $9.06 \pm 0.06$ & $4.12 \pm 0.01$ & Keck-NIRC2 \\
\hline ROXs 12 & 56022 & $K^{\prime}$ & 70 & $1783.0 \pm 1.8$ & $8.85 \pm 0.06$ & $5.02 \pm 0.01$ & Keck-NIRC2 \\
\hline ROXs 42B (b) & 55735 & $K^{\prime}$ & 108 & $1170.5 \pm 1.2$ & $269.98 \pm 0.10$ & $6.38 \pm 0.01$ & Keck-NIRC2 \\
\hline ROXs 42B (b) & 55735 & $H$ & 100 & $1170.2 \pm 1.2$ & $270.02 \pm 0.10$ & $6.89 \pm 0.02$ & Keck-NIRC2 \\
\hline ROXs 42B (b) & 55735 & $J$ & 150 & $1166 \pm 2$ & $269.74 \pm 0.16$ & $7.09 \pm 0.05$ & Keck-NIRC2 \\
\hline ROXs 42B (b) & 55735 & $L^{\prime}$ & 126 & $1172.1 \pm 1.2$ & $269.99 \pm 0.10$ & $5.70 \pm 0.04$ & Keck-NIRC2 \\
\hline ROXs 42B (b) & 56022 & $K^{\prime}$ & 260 & $1172.0 \pm 1.2$ & $270.03 \pm 0.10$ & $6.41 \pm 0.01$ & Keck-NIRC2 \\
\hline ROXs 42B (b) & 56022 & $H$ & 80 & $1170.1 \pm 1.2$ & $269.99 \pm 0.10$ & $6.84 \pm 0.05$ & Keck-NIRC2 \\
\hline ROXs 42B (b) & 56022 & $J$ & 40 & $1165.9 \pm 1.7$ & $269.47 \pm 0.10$ & $6.98 \pm 0.12$ & Keck-NIRC2 \\
\hline ROXs 42B (b) & 56022 & $K_{c}$ & 40 & $1171 \pm 2$ & $269.98 \pm 0.10$ & $6.35 \pm 0.05$ & Keck-NIRC2 \\
\hline ROXs 42B (b) & 56511 & $K^{\prime}$ & 40 & $1172.5 \pm 1.2$ & $270.25 \pm 0.10$ & $6.32 \pm 0.02$ & Keck-NIRC2 \\
\hline ROXs 42B (cc1) & 55735 & $K^{\prime}$ & 108 & $575.7 \pm 0.6$ & $223.92 \pm 0.20$ & $6.59 \pm 0.02$ & Keck-NIRC2 \\
\hline ROXs 42B (cc1) & 55735 & $H$ & 50 & $560 \pm 8$ & $224.06 \pm 0.25$ & $6.20 \pm 0.03$ & Keck-NIRC2 \\
\hline ROXs 42B (cc1) & 55735 & $L^{\prime}$ & 126 & $572 \pm 4$ & $225.0 \pm 0.5$ & $6.59 \pm 0.09$ & Keck-NIRC2 \\
\hline ROXs 42B (cc1) & 56022 & $K^{\prime}$ & 260 & $568.4 \pm 0.6$ & $225.20 \pm 0.20$ & $6.76 \pm 0.03$ & Keck-NIRC2 \\
\hline ROXs 42B (cc1) & 56511 & $K^{\prime}$ & 40 & $539.8 \pm 1.2$ & $2255.96 \pm 0.15$ & $6.93 \pm 0.04$ & Keck-NIRC2 \\
\hline ScoPMS 214 & 54636 & $K^{\prime}$ & 60 & $3079 \pm 3$ & $121.13 \pm 0.04$ & $5.78 \pm 0.02$ & Keck-NIRC2 \\
\hline ScoPMS 214 & 56022 & $K^{\prime}$ & 91 & $3071 \pm 3$ & $120.38 \pm 0.04$ & $5.77 \pm 0.05$ & Keck-NIRC2 \\
\hline ScoPMS 214 & 56032 & $K^{\prime}$ & 54 & $3071 \pm 3$ & $120.49 \pm 0.04$ & $5.84 \pm 0.01$ & Keck-NIRC2 \\
\hline ScoPMS 42b (cc1) & 54256 & $\mathrm{Br} \gamma$ & 44 & $2980 \pm 3$ & $105.41 \pm 0.05$ & $7.16 \pm 0.13$ & Keck-NIRC2 \\
\hline ScoPMS 42b (cc1) & 55392 & $K_{c}$ & 240 & $2984 \pm 3$ & $103.51 \pm 0.04$ & $7.41 \pm 0.05$ & Keck-NIRC2 \\
\hline ScoPMS 42b (cc2) & 54256 & $\mathrm{Br} \gamma$ & 44 & $4309 \pm 4$ & $332.62 \pm 0.03$ & $6.79 \pm 0.06$ & Keck-NIRC2 \\
\hline ScoPMS 42b (cc2) & 55392 & $K_{c}$ & 240 & $4362 \pm 4$ & $332.66 \pm 0.03$ & $6.76 \pm 0.02$ & Keck-NIRC2 \\
\hline ScoPMS 42b (cc3) & 54256 & $\mathrm{Br} \gamma$ & 44 & $4418 \pm 4$ & $331.77 \pm 0.03$ & $7.22 \pm 0.14$ & Keck-NIRC2 \\
\hline ScoPMS 42b (cc3) & 55392 & $K_{c}$ & 240 & $4467 \pm 4$ & $331.89 \pm 0.03$ & $7.16 \pm 0.04$ & Keck-NIRC2 \\
\hline
\end{tabular}


Table 3

(Continued)

\begin{tabular}{|c|c|c|c|c|c|c|c|}
\hline Name & $\begin{array}{c}\text { JD } \\
(-2400000)\end{array}$ & Filter & $\begin{array}{c}t_{\text {int }} \\
(\mathrm{sec})\end{array}$ & $\begin{array}{c}\rho \\
\text { (mas) }\end{array}$ & $\begin{array}{l}\text { P.A. } \\
\text { (deg) }\end{array}$ & $\begin{array}{c}\Delta m \\
(\mathrm{mag})\end{array}$ & Tel/Inst \\
\hline UScoJ1608-1935 & 54257 & $\mathrm{Br} \gamma$ & 32 & $4209 \pm 6$ & $251.36 \pm 0.13$ & $7.19 \pm 0.07$ & Keck-NIRC2 \\
\hline UScoJ1608-1935 & 56054 & $K^{\prime}$ & 60 & $4156 \pm 4$ & $252.45 \pm 0.04$ & $7.35 \pm 0.06$ & Keck-NIRC2 \\
\hline UScoJ1608-1935 & 56054 & $K^{\prime}+$ corona 600 & 40 & $4160 \pm 4$ & $252.37 \pm 0.03$ & $7.33 \pm 0.10$ & Keck-NIRC2 \\
\hline UScoJ1610-1913 & 55735 & $K^{\prime}$ & 80 & $5836 \pm 6$ & $114.00 \pm 0.02$ & $3.80 \pm 0.01$ & Keck-NIRC2 \\
\hline UScoJ1610-1913 & 55735 & $J$ & 40 & $5835 \pm 6$ & $113.98 \pm 0.02$ & $3.86 \pm 0.02$ & Keck-NIRC2 \\
\hline UScoJ1610-1913 & 55735 & $H$ & 40 & $5836 \pm 6$ & $113.99 \pm 0.02$ & $4.02 \pm 0.01$ & Keck-NIRC2 \\
\hline UScoJ1610-1913 & 55735 & $L^{\prime}$ & 80 & $5835 \pm 6$ & $114.01 \pm 0.02$ & $3.39 \pm 0.01$ & Keck-NIRC2 \\
\hline UScoJ1610-1913 & 56022 & $K^{\prime}$ & 60 & $5837 \pm 6$ & $113.94 \pm 0.02$ & $3.80 \pm 0.01$ & Keck-NIRC2 \\
\hline
\end{tabular}

Table 4

Candidate Companion Properties

\begin{tabular}{|c|c|c|c|c|c|c|c|c|c|}
\hline Name & $\begin{array}{c}M_{K^{\prime}} \\
(\mathrm{mag})\end{array}$ & $\begin{array}{c}m_{J} \\
(\mathrm{mag})\end{array}$ & $\begin{array}{c}m_{H} \\
(\mathrm{mag})\end{array}$ & $\begin{array}{c}m_{K^{\prime}} \\
(\mathrm{mag})\end{array}$ & $\begin{array}{c}m_{L^{\prime}} \\
(\mathrm{mag})\end{array}$ & 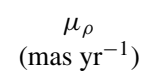 & 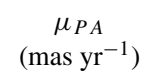 & $\begin{array}{c}\rho \\
(\mathrm{AU})\end{array}$ & $\begin{array}{c}M \\
\left(M_{\text {Jup }}\right)\end{array}$ \\
\hline \multicolumn{10}{|c|}{ New Companions } \\
\hline ROXs 12 & $8.93 \pm 0.19$ & $16.64 \pm 0.07$ & $15.37 \pm 0.08$ & $14.32 \pm 0.06$ & $12.55 \pm 0.09$ & $3.4 \pm 2.0$ & $-6.5 \pm 1.9$ & $210 \pm 20$ & $16 \pm 4$ \\
\hline ROXs 42B (b) & $9.62 \pm 0.19$ & $16.99 \pm 0.07$ & $15.88 \pm 0.06$ & $15.01 \pm 0.05$ & $14.15 \pm 0.09$ & $0.2 \pm 1.1$ & $-0.7 \pm 1.6$ & $140 \pm 10$ & $10 \pm 4$ \\
\hline \multicolumn{10}{|l|}{ Known Companions } \\
\hline DH Tau & $8.34 \pm 0.24$ & $15.69 \pm 0.07$ & $14.88 \pm 0.07$ & $14.15 \pm 0.07$ & $12.93 \pm 0.08$ & $0.2 \pm 0.2$ & $-1.0 \pm 0.3$ & $340 \pm 30$ & $18 \pm 4$ \\
\hline UScoJ1610-1913 & $7.00 \pm 0.23$ & $13.89 \pm 0.08$ & $13.28 \pm 0.07$ & $12.80 \pm 0.06$ & $12.09 \pm 0.09$ & $1.8 \pm 1.8$ & $-1.2 \pm 1.6$ & $850 \pm 90$ & $70 \pm 10$ \\
\hline GSC 6214-210 & $9.15 \pm 0.23$ & $16.30 \pm 0.08$ & $15.55 \pm 0.07$ & $14.95 \pm 0.06$ & $13.83 \pm 0.09$ & $0.4 \pm 0.6$ & $-1.7 \pm 0.6$ & $320 \pm 30$ & $16 \pm 1$ \\
\hline \multicolumn{10}{|c|}{ Unassociated Objects } \\
\hline $2 \mathrm{M} 04153916$ & $\ldots$ & & & $14.91 \pm 0.07$ & & & & & \\
\hline HD 27659 & $\ldots$ & $14.31 \pm 0.07$ & $14.03 \pm 0.07$ & $14.09 \pm 0.07$ & $14.37 \pm 0.10$ & $-5.5 \pm 4.2$ & $7.8 \pm 4.2$ & & \\
\hline ScoPMS 42b (cc2) & $\ldots$ & $\ldots$ & $\ldots$ & $16.39 \pm 0.07$ & $\ldots$ & $17.0 \pm 1.8$ & $0.9 \pm 1.1$ & & \\
\hline ScoPMS 42b (cc3) & $\ldots$ & $\ldots$ & $\ldots$ & $16.79 \pm 0.07$ & $\ldots$ & $15.8 \pm 1.8$ & $2.9 \pm 1.1$ & & \\
\hline GSC 06793-00994 & $\ldots$ & $\ldots$ & $\ldots$ & $16.41 \pm 0.06$ & $\ldots$ & $17.4 \pm 2.6$ & $16.2 \pm 3.2$ & & \\
\hline GSC 06794-00156 & $\ldots$ & $\ldots$ & $\ldots$ & $16.51 \pm 0.07$ & $\ldots$ & $7.3 \pm 3.8$ & $14.6 \pm 4.3$ & & \\
\hline DoAr 22 & $\ldots$ & $15.95 \pm 0.07$ & $\ldots$ & $14.96 \pm 0.06$ & $\ldots$ & $16.0 \pm 2.1$ & $24.1 \pm 2.2$ & & \\
\hline ScoPMS 214 & $\ldots$ & $\ldots$ & $\ldots$ & $13.57 \pm 0.06$ & $\ldots$ & $-1.1 \pm 0.8$ & $-8.6 \pm 0.6$ & & \\
\hline ROXs 42B (cc1) & $\ldots$ & $\ldots$ & $15.22 \pm 0.07$ & $15.46 \pm 0.06$ & $15.04 \pm 0.12$ & $-9.1 \pm 1.1$ & $14.4 \pm 3.2$ & & \\
\hline
\end{tabular}

Notes. Each photometry measurement reflects the weighted mean of all measurements in Tables 2 and 3, except those that are footnoted as having been explicitly excluded. The minimum uncertainty at an epoch is assumed to be $\sigma=0.05$ mag (due to stellar variability of the primary). The uncertainties here also reflect the uncertainty in the primary star magnitudes taken from 2MASS and WISE, as well as the color-SpT relation used to convert WISE W1 to MKO $L^{\prime}$. Stellar variability at those epochs also imposes a systematic uncertainty of $\sigma=0.05$ mag. The magnitudes reported here have not been corrected for extinction, but we have de-extincted or dereddened using the $A_{V}$ inferred for each corresponding primary (Table 1) before computing masses or colors (i.e., Figures 7-10). The uncertainties in projected separations are driven entirely by the uncertainty in distance and hence have the same fractional uncertainty as the quoted values (Section 2.3 ).

We obtained further observations of the FW Tau system in 2008 and 2011 and, as we show in Figure 1 (left), the candidate companion has a significant (but quite faint) NIR counterpart. The multi-epoch astrometry shown in Figure 1 (right) demonstrates that the candidate is indeed a comoving companion, with a net relative motion of $1.7 \pm 0.9$ mas $\mathrm{yr}^{-1}$ $\left(\sim 1.1 \mathrm{~km} \mathrm{~s}^{-1}\right)$ with respect to the primary of the central pair. Based on its dereddened absolute magnitude of $M_{K^{\prime}}=$ $9.28 \pm 0.24$, we estimate a companion mass of $10 \pm 4 M_{\text {Jup }}$ for system ages of 1-5 Myr (Chabrier et al. 2000). The extremely red color of the companion (dereddened $J-K^{\prime}=1.73 \pm 0.13$ ) is consistent with this identification, especially given the low line-of-sight reddening to $\mathrm{FW}$ Tau $\mathrm{AB}\left(A_{V}=0.4\right.$; Table 1$)$. This very red $J-K^{\prime}$ color is typically only seen among field dwarfs for the reddest late- $\mathrm{L}$ dwarfs but, as was noted by Allers et al. (2010), young objects often seem to be redder than field counterparts of similar temperature. Theoretical models predict that the companion should have a temperature of $T_{\text {eff }} \sim$ 1900-2100 K and hence a spectral type of $\sim$ L1-L2. Given its bound nature and apparently planetary mass, we hereafter denote the companion as "FW Tau (AB) b" or "FW Tau b" for simplicity.

The significant $\mathrm{H} \alpha$ emission from FW Tau b, combined with the possible presence of a disk somewhere in the system, indicate a high likelihood that it hosts a (circumplanetary?) disk like that of GSC 6214-210 b (Bowler et al. 2011). The presence of this disk also introduces some ambiguity regarding the intrinsic luminosity of the companion, as it could be a stellar or BD 

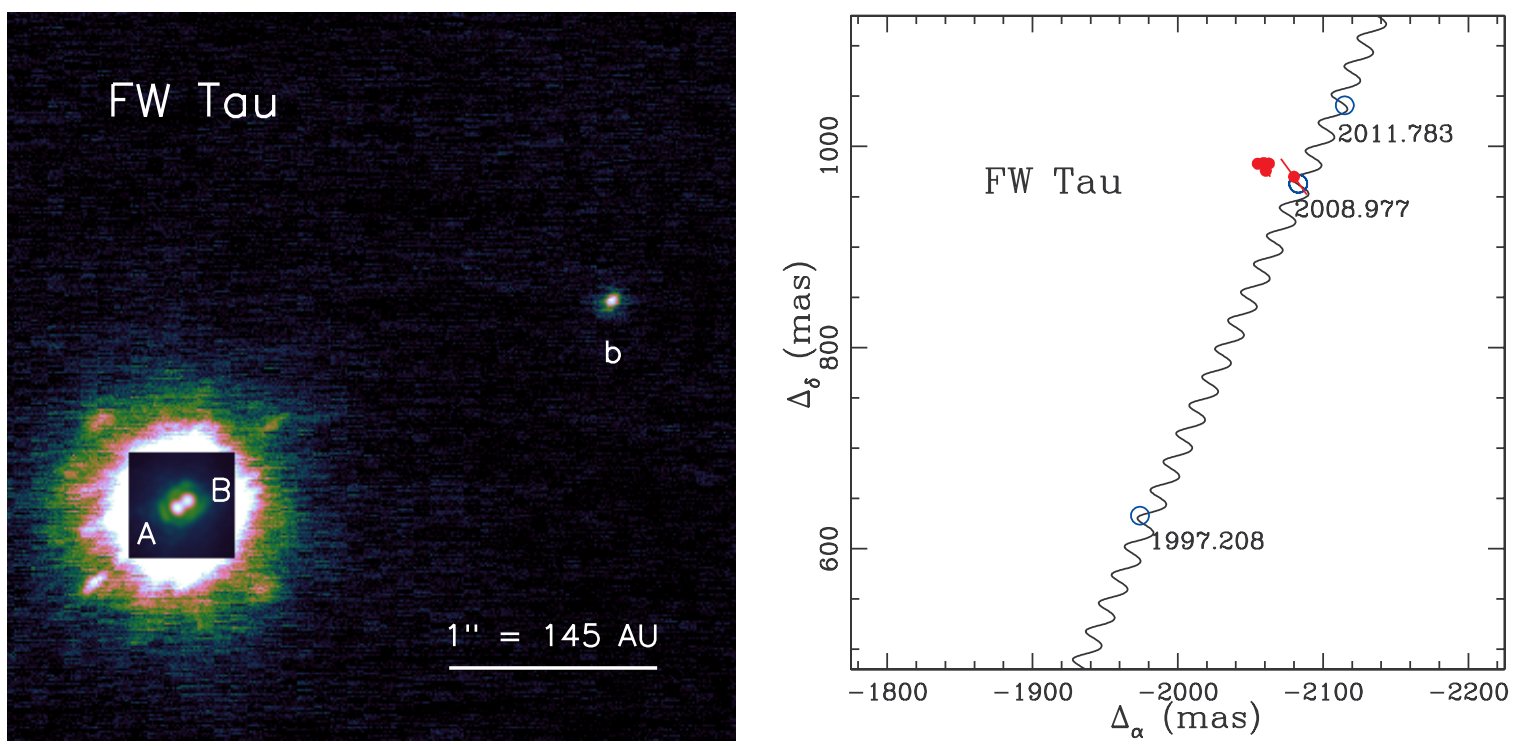

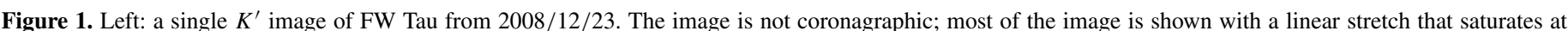

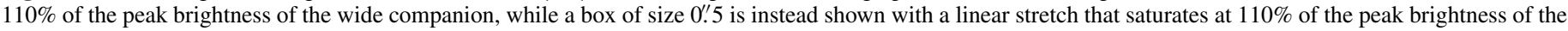

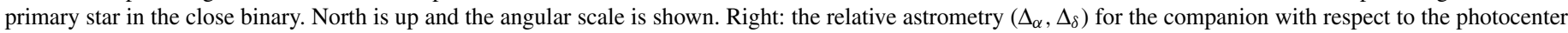

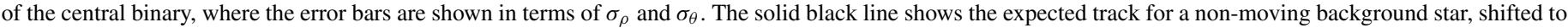
minimize the weighted residuals with respect to the expected positions at each epoch of observation (blue circles).

(A color version of this figure is available in the online journal.)

companion with an edge-on disk (like HV Tau C or HK Tau B; Stapelfeldt et al. 1998; Duchêne et al. 2010). However, the morphology argues against this explanation. Stellar edgeon disks show a characteristic central dark lane that separates the light reflecting from the two surfaces of the disk and we see only a single unresolved point source.

There are two known substellar hosts of edge-on disks, IRAS 04325+2402 C (Hartmann et al. 1999; Scholz et al. 2008) and 2MASS J04381486+2611399 (Luhman et al. 2007), both with $\operatorname{SpT} \sim \mathrm{M} 7.25$. IRAS $04325+2402 \mathrm{C}$ has a mass of $M<0.1 M_{\text {sun }}$, although the NIR spectrum is heavily veiled and a more precise mass determination is difficult. HST images clearly show the characteristic dark lane seen for higher-mass edgeon disks, indicating that at least some substellar edge-on disk systems follow this morphology. 2MASS J04381486+2611399 is an $\mathrm{M} 7.25 \mathrm{BD}\left(M \sim 50 M_{\mathrm{Jup}}\right)$ that also appears to host an edge-on disk, but is more easily characterized from its scattered light spectrum because its inner disk is cleared and hence there is no veiling or NIR excess. HST images show a bipolar outflow emerging from the $\mathrm{BD}$, but there is not a clear dark lane. However, it is $>2$ mag brighter than FW Tau b in $K^{\prime}$. Assuming similar amounts of attenuation in the $K^{\prime}$ flux and neglecting possible NIR excesses in FW Tau b, then the 1 Myr DUSTY models still indicate that FW Tau b would have $M<15 M_{\text {Jup }}$ if seen in a similar geometry. Nonetheless, a dispositive result would require spectroscopic detection of photospheric features that indicate the $T_{\text {eff }}$ of the central object.

\subsection{2. $R O X s 42 B b$}

ROXs 42B AB is a little-studied member of the Ophiuchus complex, located $\sim 2^{\circ}$ east of its core. ROXs $42 \mathrm{~B}$ was identified as a close binary system by Simon et al. (1995) and Ratzka et al. (2005); the latter survey measured a flux ratio of $\Delta K \sim 1.1$ and a projected separation of $\rho=83$ mas $(\sim 10 \mathrm{AU})$. As we will report in a future work, our own observations with nonredundant aperture-mask interferometry have not recovered this companion at a projected separation of $\rho>15$ mas, suggesting that orbital motion has carried it inward over the past decade. The (unresolved) system was studied with optical spectroscopy by Bouvier \& Appenzeller (1992), who determined a spectral type of M0 and identified it as a young star based on the presence of weak $\mathrm{H} \alpha$ emission $(\mathrm{EW}[\mathrm{H} \alpha]=-1.7 \AA)$, X-ray emission, and possible lithium absorption. Models suggest individual component masses of $0.89 \pm 0.08 M_{\odot}$ and $0.36 \pm 0.04 M_{\odot}$, respectively, and a corresponding isochronal age of $6.8_{-2.3}^{+3.4} \mathrm{Myr}$. The system has no excess in $W 3$ and no counterpart in $W 4$ from WISE observations, no radio counterpart at $1.3 \mathrm{~mm}$ $\left(F_{v}<45 \mathrm{mJy} ; M_{\text {disk }}<9 M_{\text {Jup }}\right.$; Andrews \& Williams 2007), and $\mathrm{H} \alpha$ emission consistent with most WTTSs and hence there is no evidence that it hosts an optically thick protoplanetary disk.

The binary survey by Ratzka et al. (2005) also identified a faint candidate companion at a much wider projected separation $\left(\sim 1^{\prime \prime}\right.$. 1; $\sim 140$ AU) in the shift-and-added stack of their speckle data. However, it has been neglected in the subsequent literature, so we observed the system in 2011 and 2012 to confirm its existence (Figure 2, left) and test for common proper motion (Figure 2, right). Our observations recovered the candidate companion, but as can be seen in Figure 2 (left), they also revealed another candidate companion even closer to ROXs 42B ( $\rho=0$ 0.55; $70 \mathrm{AU})$.

As we show in Figure 2 (right), the outer candidate is indeed a comoving companion to ROXs $42 \mathrm{~B}$, with a relative motion of

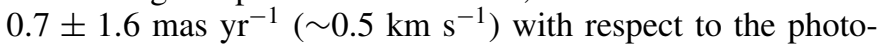
center of the central binary. Based on its dereddened absolute magnitude of $M_{K^{\prime}}=9.39 \pm 0.19$, we estimate a companion mass of $10 \pm 4 M_{\text {Jup }}$ for system ages of 1-5 Myr (Chabrier et al. 2000). The companion has a luminosity and colors very similar to FW Tau b, so we expect similar atmospheric and bulk properties. Given its bound nature and apparently planetary mass, we hereafter denote the companion (with some regret regarding the nomenclature) as "ROXs 42B (AB) b" or "ROXs 42B b" for simplicity. 

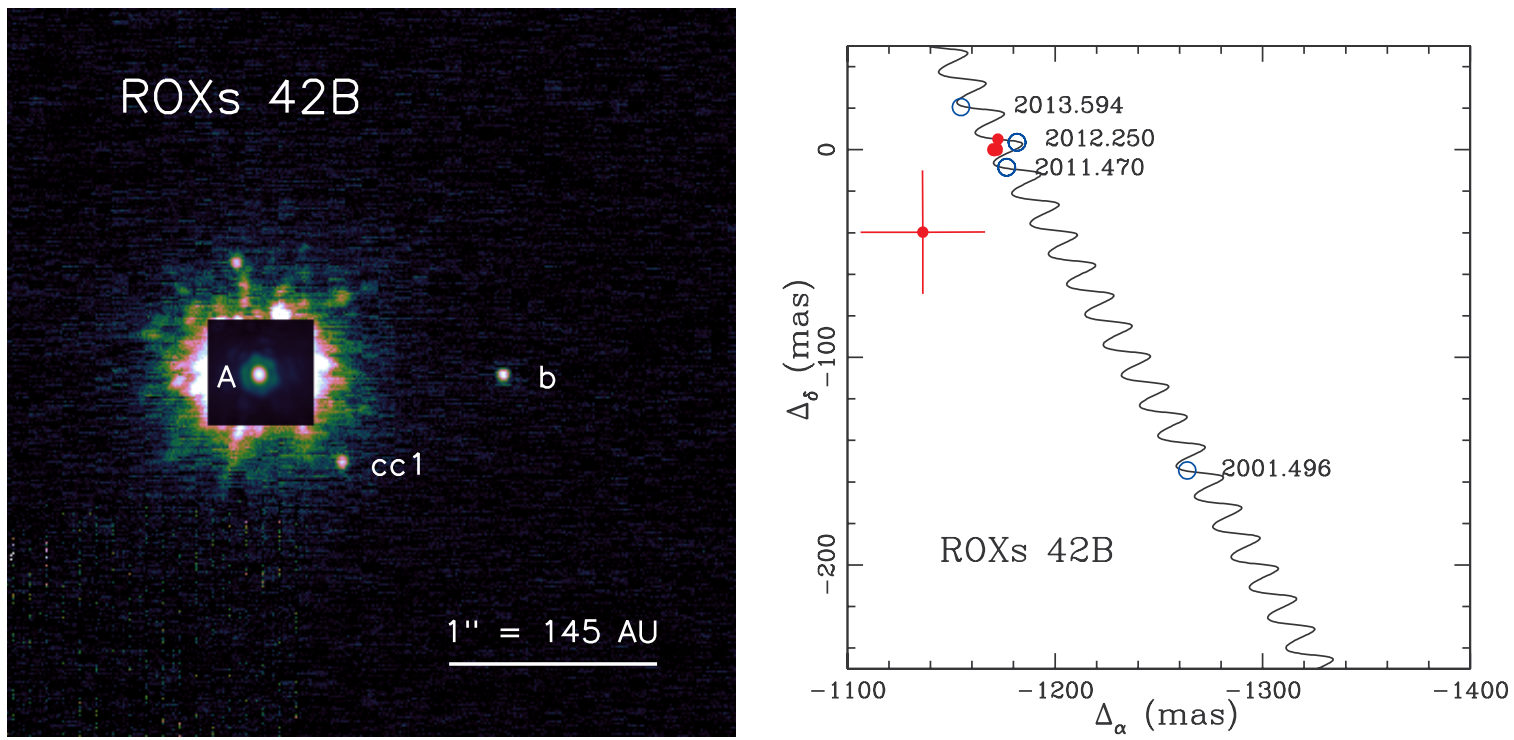

Figure 2. As in Figure 2, but for ROXs 42B b. The image is from 2011 June 22. A long-term superspeckle can be seen above ROXs 42B A; it and other such features can be recognized and rejected by their chromatic behavior in our multi-wavelength data.

(A color version of this figure is available in the online journal.)

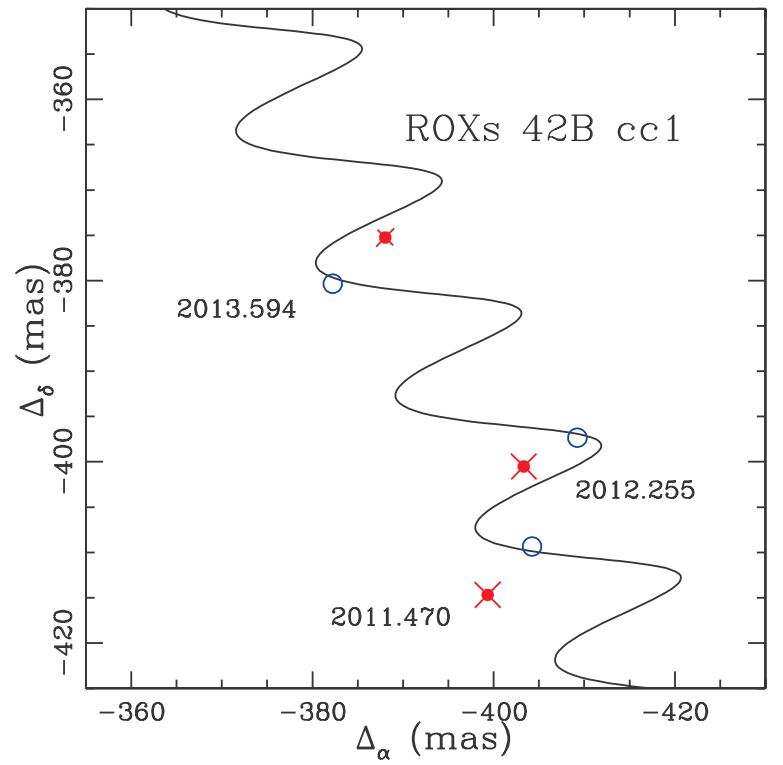

Figure 3. As in the right panel of Figure 3, but for ROXs 42B cc1. The observed motion is small and the probability of finding a background star at $\rho<1^{\prime \prime}$ is small, but given the broad agreement with the track expected for a non-moving background star, then the most likely conclusion is that $\mathrm{cc} 1$ is not associated with ROXs 42B. The level of disagreement with non-movement is within the uncertainty in the proper motion of ROXs 42B A $\left( \pm 3.4\right.$ mas yr$\left.^{-1}\right)$.

(A color version of this figure is available in the online journal.)

In Figure 3, we show the corresponding proper motion diagram for the inner candidate. The nature of this object is less certain; the proximity to the bright primary leaves its colors and astrometry less reliable and the larger expected orbital motion

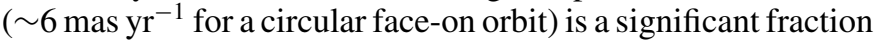
of the absolute proper motion of the system. Nonetheless, the measured astrometry agrees very well with the track expected for a non-moving background star, with a total relative motion of $17 \pm 3$ mas $\mathrm{yr}^{-1}$, and its colors appear to be quite blue $\left(H-K^{\prime}=-0.21 \pm 0.11, K^{\prime}-L^{\prime}=0.09 \pm 0.14\right)$. We therefore strongly prefer the background hypothesis over the bound companion hypothesis for this object and we provisionally designate it as a background star (hereafter, "ROXs 42B cc1").

\subsection{3. $\operatorname{ROXs} 12 b$}

ROXs 12 is another neglected member of the Ophiuchus complex, located $\sim 1^{\circ}$ south of its core. Bouvier \& Appenzeller (1992) determined a spectral type of M0 and identified it as a young star based on the presence of weak $H \alpha$ emission $(\mathrm{EW}[\mathrm{H} \alpha]=-1.2 \AA), \mathrm{X}$-ray emission, and lithium absorption. The HR diagram position of ROXs 12 corresponds to a mass of $0.87 \pm 0.08 M_{\odot}$ and an age of $7.6_{-2.5}^{+4.1}$ Myr. A significant excess in the WISE $W 3$ and $W 4$ filters indicates that the system hosts an optically thick protoplanetary disk, although the nondetection of emission at $1.3 \mathrm{~mm}$ by (Andrews \& Williams 2007) $\left(F_{v}<\right.$ $19 \mathrm{mJy})$ suggests that the disk is not very massive $\left(M_{\text {disk }}<\right.$ $\left.4 M_{\text {Jup }}\right)$. Also, the $\mathrm{H} \alpha$ line strength is consistent with most WTTSs and much lower than is seen for typical accreting stars. Ratzka et al. (2005) found no evidence of any close $\left(\lesssim 1^{\prime \prime}\right)$ binary companions from speckle interferometry, a result confirmed and extended to much smaller radii by our observations with nonredundant aperture-mask interferometry (A. Cheetham et al., in preparation). Ratzka et al. (2005) did note a faint candidate companion at a projected separation of $\sim 1^{\prime \prime}$.7 (210 AU) in the shift-and-added stack of their speckle interferometry data. However, it subsequently has been neglected in the literature.

We obtained additional observations of ROXs 12 in 2011 and 2012, confirming the existence of the candidate companion (Figure 4, left). The multi-epoch astrometry demonstrates that the candidate companion has a proper motion similar to that of ROXs 12, with a relative motion of $7 \pm 3$ mas $\mathrm{yr}^{-1}$ $\left(4.2 \pm 1.6 \mathrm{~km} \mathrm{~s}^{-1}\right)$ (Figure 4, right). The companion has dereddened $J-H$ and $H-K^{\prime}$ colors very similar to those of ROXs 42B b, but is significantly redder at longer wavelengths, with $K^{\prime}-L^{\prime}=1.46 \pm 0.11$. While the measurement of comovement is more discrepant than for the other two new PMCs, our results rule out non-movement at high significance. If the candidate was unassociated, it therefore would be a nearby field star and the red colors of this candidate clearly distinguish it from the field population. Based on its absolute magnitude 

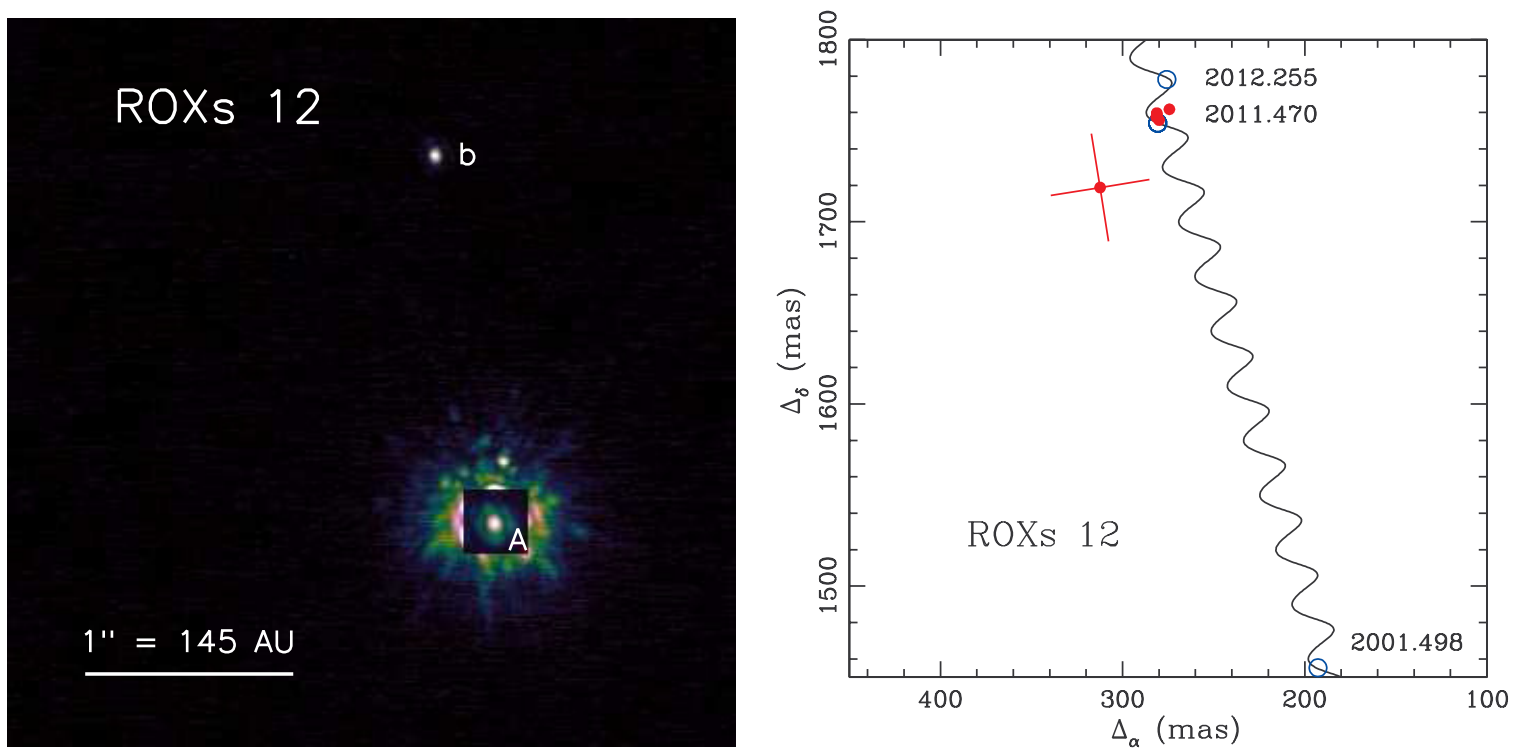

Figure 4. As in Figure 2, but for ROXs 12. The image is from 2011 June 22.

(A color version of this figure is available in the online journal.)

of $M_{K^{\prime}}=8.42 \pm 0.19$, we estimate a companion mass of $16 \pm 4 M_{\text {Jup }}$ for system ages of 1-5 Myr (Chabrier et al. 2000). As a bound companion of approximately planetary mass, we denote the companion as "ROXs 12 b."

As for FW Tau b, the presence of a disk in the system (determined from the WISE W3 and W4 photometry) could indicate that we have observed a binary companion that is obscured by an edge-on disk. Given the lack of accretion onto the apparent primary of the system, the argument must be given special consideration for ROXs $12 \mathrm{~b}$. However, as we discussed in Section 4.1.1, the point source morphology seen in our NIR images presents the same argument against this interpretation as for FW Tau b. The system is also quite luminous in $W 3$ $\left(m_{W 3}=5.99\right)$, indicating that the companion would need to be massive and intrinsically luminous. The disk therefore is most likely associated with the primary star and the presence or absence of a disk around the companion cannot be inferred from the existing data.

\subsection{Unassociated Background Sources}

As we show in Figure 5, six of the candidate companions that we have identified have relative motions that are similar to those expected for a non-moving background star. We therefore denote these apparent background sources as DoAr $22 \mathrm{cc} 1$, GSC 06793-00994 cc1, GSC 06794-00156 cc1, PDS 70 cc1, UScoJ1608-1935 cc1, and ScoPMS 42b cc1 and have referred to them as such in this work. In most cases, the agreement with comovement is not exact, indicating that the candidate companion has a non-zero absolute proper motion, but these

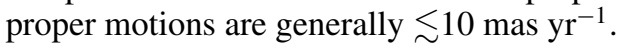

In Figure 6, we show the relative motions of four more candidates that appear to be moving with absolute motions of similar magnitude as (but in a different direction than) their host stars, indicating that they are likely not as distant as the candidates listed above. One target (hereafter denoted GSC 06191-00019 cc1) follows a uniform vector that is perpendicular (and of similar magnitude) to that of its candidate host star. Another target (ScoPMS 214) actually appears to be nearly comoving with its candidate host star, but past spectroscopic observations indicate that it is likely a field dwarf in the solar neighborhood (Metchev \& Hillenbrand 2009). Finally, ScoPMS $42 \mathrm{~b}$ hosts a pair of faint sources (separated by $\sim 150$ mas) that appear to be comoving with each other, but not comoving with the candidate host. These sources were discovered (but not individually resolved) by Köhler et al. (2000), who found relative astrometry that is also consistent with the tracks that we observe. We infer that these objects (denoted ScoPMS 42b cc2 and ScoPMS 42b cc3) are a binary system comprised of two similar-mass field dwarfs.

Finally, there are two additional targets for which we cannot determine a proper motion, but which we can classify as unassociated by other criteria. Both candidate companions (2M0415+2813 cc1 and HD 27659 cc1) were identified when we conducted snapshot AO imaging (in preparation for future deep observations) of newly identified Taurus members identified by Luhman et al. (2009) and Rebull et al. (2010). We concluded from the snapshot imaging that 2M0415+2813 cc1 was most likely a background galaxy, based on an elliptical elongation of its PSF that is not aligned with the P.A. to the host star (i.e., anisoplanatism) or with the elevation angle (i.e., windshake or dispersion). Subsequent to this determination, images from SDSS DR9 (Ahn et al. 2012) detected the candidate in the $g^{\prime}$ and $r^{\prime}$ filters with a contrast of 4-5 mag. Since late-M dwarfs have colors $g^{\prime}-K^{\prime} \gtrsim 10$ (Kraus \& Hillenbrand 2007b), we conclude the candidate companion is indeed an unassociated background source. The other candidate companion, HD $27659 \mathrm{cc} 1$, has low-quality astrometry from the discovery observations since the candidate fell on a high point in NIRC2's spatially correlated read-noise pattern; even though we have imaging observations from separate seasons, a test of common proper motion is inconclusive. However, the color of the companion is quite blue $\left(J-L^{\prime}=0.12 \pm 0.13\right)$, so it is unlikely to be a PMC. The proper motion of HD 27659 is also inconsistent with Taurus membership, so even though its position and distance place it within the Taurus molecular cloud (Kenyon et al. 1994), we suggest that the system is unlikely to be young.

\subsection{Updated Photometry and Astrometry for Known PMCs}

We also have observed five known PMCs in order to obtain more or better colors than were available in the literature, as well 

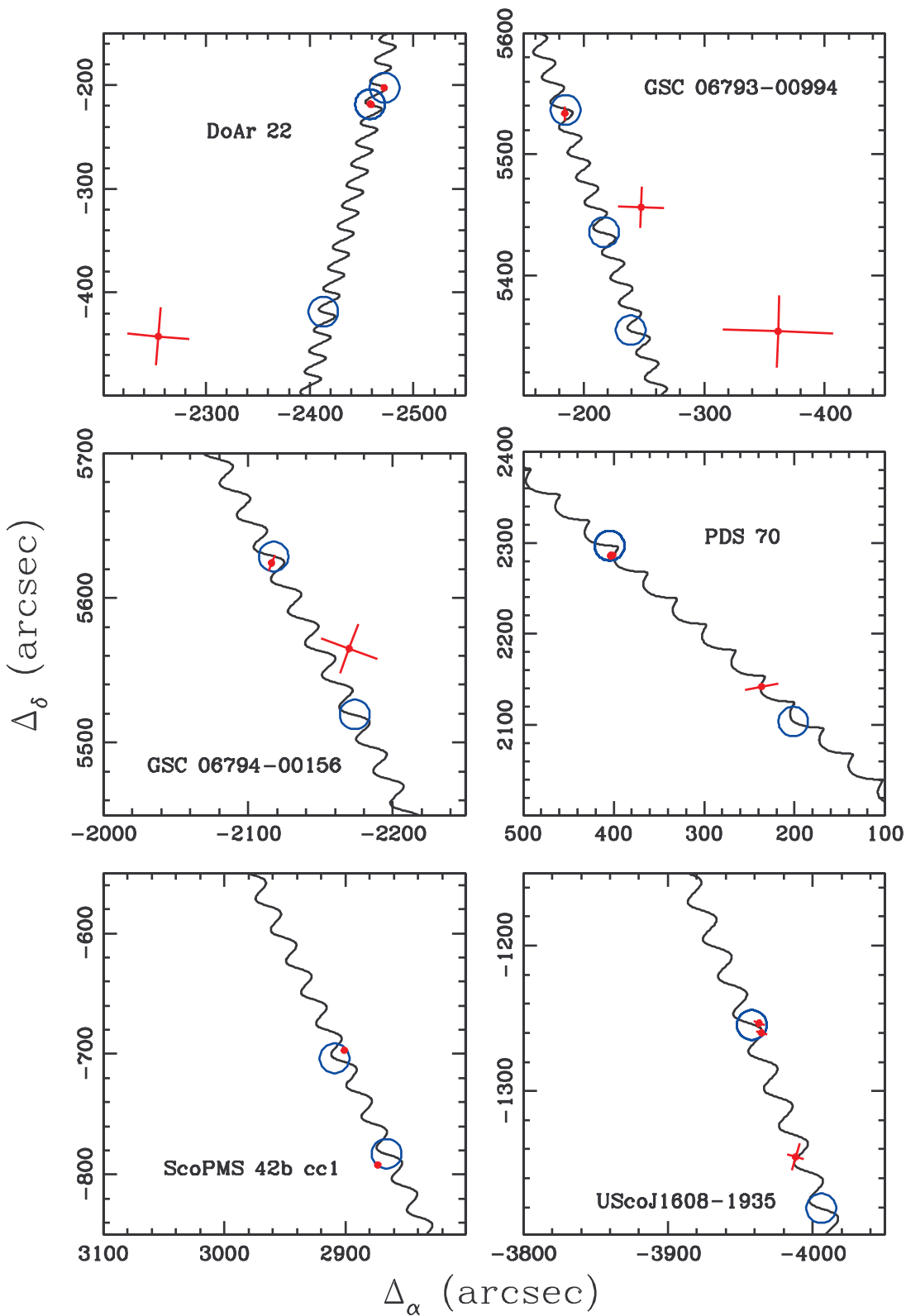

Figure 5. Relative astrometry $\left(\Delta_{\alpha}, \Delta_{\delta}\right)$ for six candidate companions with respect to the photocenter of their candidate host star, where the error bars are shown in terms of $\sigma_{\rho}$ and $\sigma_{\theta}$. The solid black line shows the expected track for a non-moving background star, shifted to minimize the weighted residuals with respect to the expected positions at each epoch of observation (blue circles). In all cases, the track of the candidate companion falls close to the non-moving track, suggesting that these candidates are unassociated, distant background objects with small proper motions.

(A color version of this figure is available in the online journal.)

as to extend the time baseline in confirming common proper motion. While their identity was not in doubt, we still have analyzed the literature data and our new observations in the same way as for the candidate PMCs (Sections 4.1-4.2). All are once again confirmed to be associated and we did not find any photometric measurements that contradict measurements in the literature.

GQ Lup continues to show significant evidence of orbital motion in the radial and tangential directions, as first pointed out by Neuhäuser et al. (2008). None of the other targets show clear evidence of motion at more than $\sim 3 \sigma$, which is not a sufficient criterion for significance among such a large sample of measurements. However, the circular orbital velocities at the observed separations ( $\sim 100-300 \mathrm{AU})$ are typically $1-2 \mathrm{~km} \mathrm{~s}^{-1}$ or $1-2$ mas $r^{-1}$. We therefore expect that these measurements will achieve statistical significance in the near future, especially with dedicated campaigns that use a single instrument/filter and control or minimize systematic sources of error such as differential chromatic refraction and tilt jitter.

\section{THE COLORS OF WIDE PMCs}

Observations of free-floating young BDs with mid-M to midL spectral types show that they follow distinct color-SpT sequences from their older (and more massive) main-sequence counterparts. In particular, young BDs show systematically redder $J-K^{\prime}$ and $K^{\prime}-L^{\prime}$ colors (e.g., Allers et al. 2010), trends that are often attributed to larger dust abundances or low surface gravities. If PMCs follow the same trend, then it would indicate a similar bulk composition and hence possibly a similar formation mechanism. Significant differences in colors could indicate either a different composition (i.e., from formation in a 


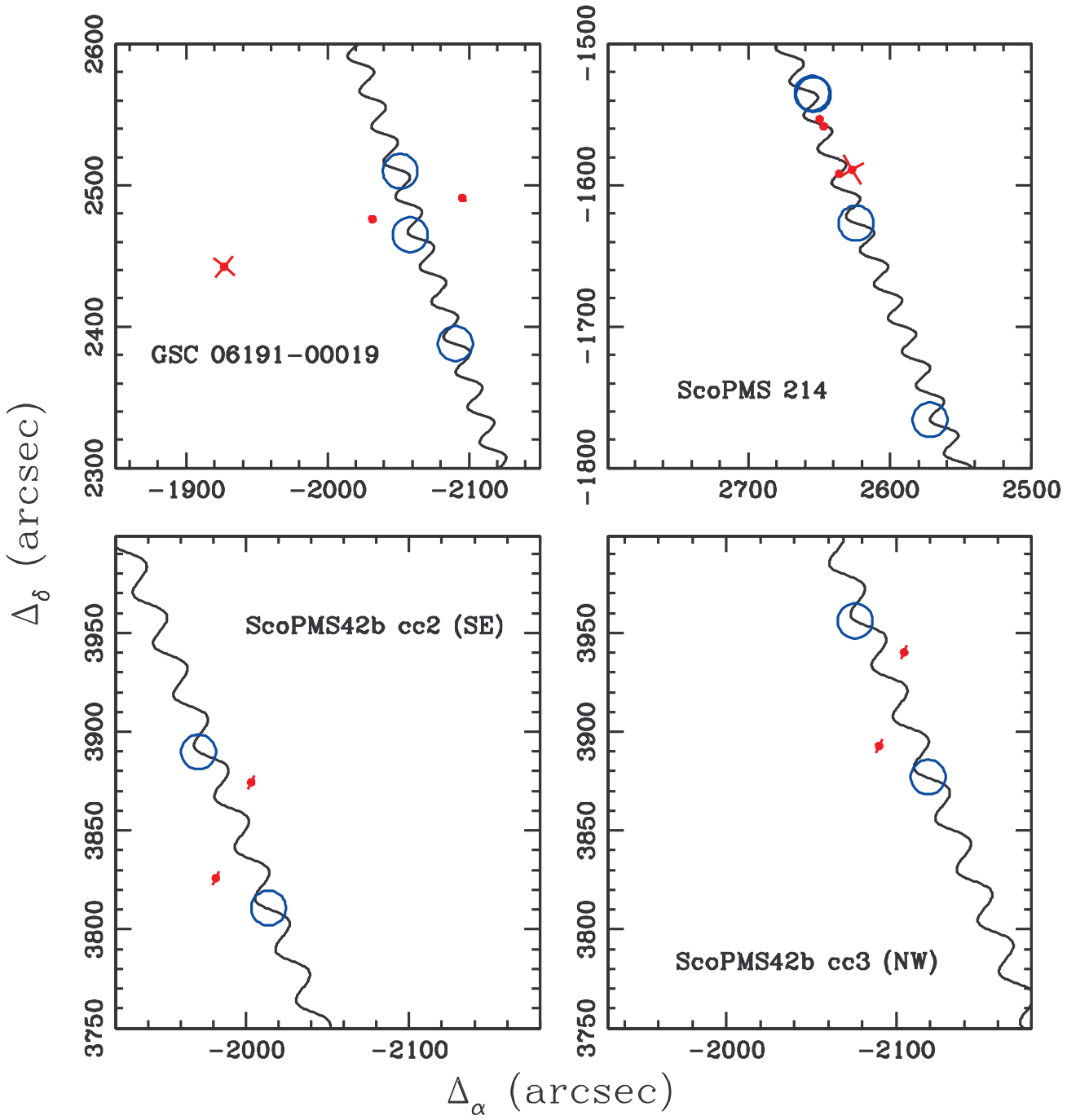

Figure 6. As in Figure 5, but for four candidate companions that display significant motion with respect to either the comoving or non-moving case.

(A color version of this figure is available in the online journal.)

chemically differentiated disk) or the presence of circum(sub)stellar phenomena like disks, outflows, and accretion.

In Figures 7 and 8, we show $\left(K^{\prime}, J-K^{\prime}\right)$ and $\left(K^{\prime}, H-K^{\prime}\right)$ color-magnitude diagrams (CMDs) for our sample of PMCs, as well as reference populations of dereddened objects in Upper Sco $(\tau=5-10 \mathrm{Myr})$ and Taurus $(\tau=1-5 \mathrm{Myr})$. We also show the predictions of the 5 Myr BCAH98, DUSTY, and COND model tracks. In both cases, the PMCs in our sample are consistent with the color/luminosity sequence and intrinsic spread for the young sequence. The young sequence seems to transition from the BCAH98 models to the DUSTY models at $J-K \sim 1.1-1.3$, as do the PMCs. However, there is only a modest level of agreement with the DUSTY models at $M_{K} \gtrsim 8$. At $8<M_{K}<10$, the young sequence and most of the PMCs sit well above the 5 Myr DUSTY track, implying that the models underestimate luminosities in this regime. However, the faintest PMC (1RXSJ1609) has nearly the same color as more luminous objects and falls below the DUSTY track. This object could represent the point where the $J-K^{\prime}$ color saturates at a maximum value (e.g., Allers et al. 2010) and begins to transition toward the COND tracks.

In general, the PMCs from younger regions (Taurus, Ophiuchus, and Lupus) sit higher on the CMD than those from Upper Sco, indicating that part of the scatter in observed PMC properties might be due to evolution. However, the properties of the primary stars do not always follow this trend. The isochronal ages for ROXs 12 and ROXs 42B are similar to those of GSC 6214-210 and 1RXSJ1609, even though they are nominally members of a younger population. This discrepancy could mean that the spectral types from the literature (which typically date to $\lesssim 1990$ ) need to be repeated with better instruments or reclassified with modern spectral classification systems.

In Figure 9, we show the $\left(J-H, H-K^{\prime}\right)$ color-color diagram for our sample. Following the format of Figures 7 and 8 , we show the BCAH98 and DUSTY models and our reference populations; we also add a sample of young $\mathrm{M} / \mathrm{L}$ field dwarfs (Allers \& Liu 2013) to illustrate the likely extension of the Taurus and Upper Sco sequences, which have no members later than L2. As for the CMDs, the PMCs largely fall along the young sequence. However, several objects fall redder than the young sequence in $H-K^{\prime}$, suggesting a possible difference in their properties.

Finally, in Figure 10 we show a corresponding $\left(J-K^{\prime}\right.$, $K^{\prime}-L$ ) color-color diagram for our sample. Past observations of these objects have found very red $K^{\prime}-L^{\prime}$ colors, significantly exceeding those seen for main-sequence field dwarfs. Intriguingly, we find that five of the seven PMCs with $M<30 M_{\text {Jup }}$ are redder than even the young sequence. Ireland et al. (2011) 


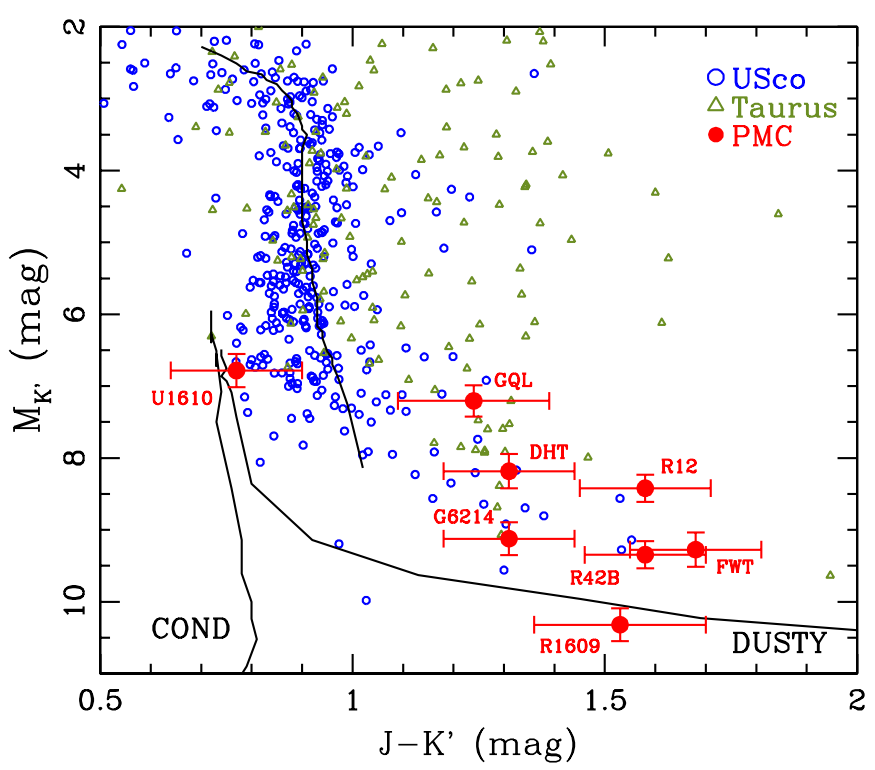

Figure 7. Color-magnitude diagram showing dereddened colors $\left(J-K^{\prime}, M_{K}^{\prime}\right)$ for our sample of wide PMCs (red filled circles). We also show a representative sample of free-floating members of Upper Sco (blue circles) and Taurus (magenta triangles). The Upper Sco sample was adopted from the census by (Kraus \& Hillenbrand 2007a), supplemented by Slesnick et al. (2008) and Lodieu et al. (2008), and dereddened for an average extinction of $A_{V}=1$. The Taurus sample was adopted from Luhman et al. (2010) and Rebull et al. (2010), dereddened individually with values from the references therein, and screened of binaries as described by Kraus et al. (2011) and Kraus \& Hillenbrand (2012). Finally, we also show the 5 Myr Lyon evolutionary models of Baraffe et al. (1998), Chabrier et al. (2000), and Baraffe et al. (2003). The PMCs follow the Upper Sco sequence in transitioning from the BCAH98 track to the DUSTY track. Once in the DUSTY regime $\left(M_{K}>8\right)$, the sequence appears to decline steeply; the brighter PMCs and Upper Sco members are overluminous for their color, while 1RXSJ1609 is underluminous and not significantly redder, indicating a possible transition toward the COND track.

(A color version of this figure is available in the online journal.)

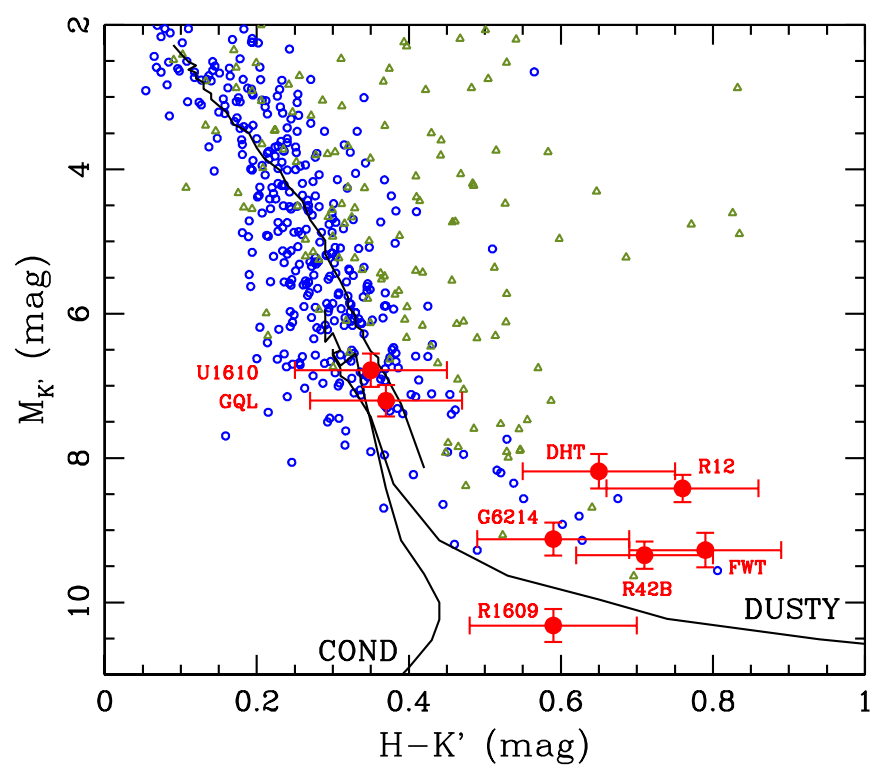

Figure 8. As in Figure 7, but showing dereddened colors $\left(H-K^{\prime}, M_{K}^{\prime}\right)$. As in Figure 8, most of the PMCs in the DUSTY regime are overluminous, while 1 RXSJ1609 is underluminous and might be trending toward the COND (dustfree) sequence.

(A color version of this figure is available in the online journal.)

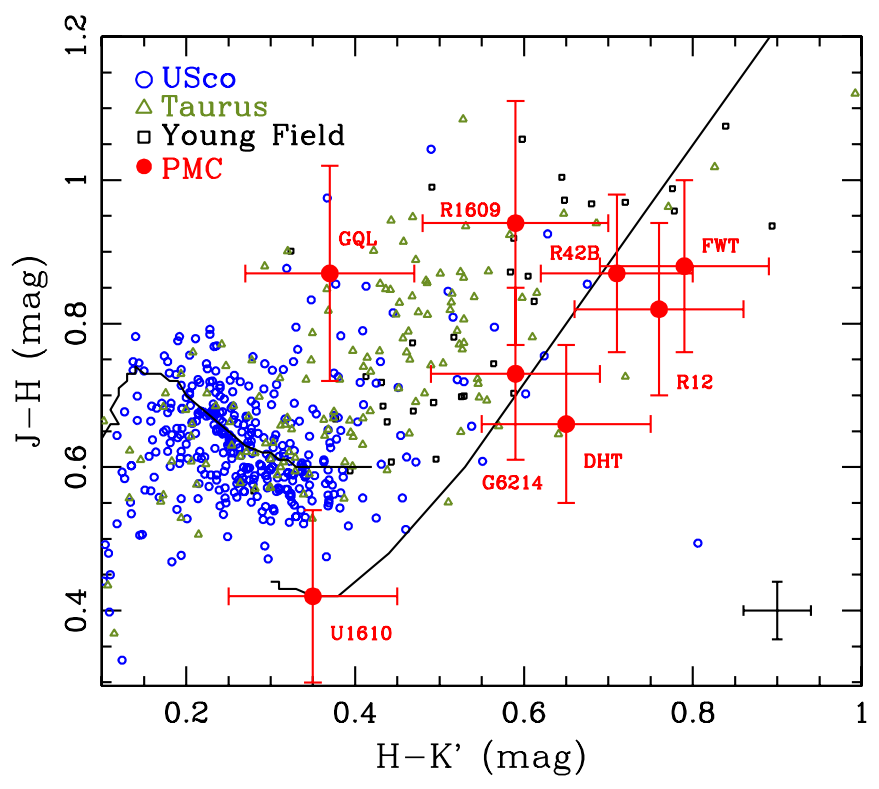

Figure 9. Color-color diagram showing dereddened colors $\left(H-K^{\prime}, J-H\right)$ for our sample of wide PMCs (red filled circles). We also show the reference populations described in Figure 7 (Upper Sco in blue and Taurus in magenta), as well as a sample of young field M/L dwarfs (black squares; Allers \& Liu 2013) that is meant to extend the young sequence past the current limits of star-forming regions. We also show the 5 Myr Lyon models of Baraffe et al. (1998) and Chabrier et al. (2000). The PMCs and Upper Sco members follow the same $\left(H-K^{\prime}, J-H\right)$ track as the models, although several PMCs are marginally redder in $H-K^{\prime}$ than the young sequence.

(A color version of this figure is available in the online journal.)

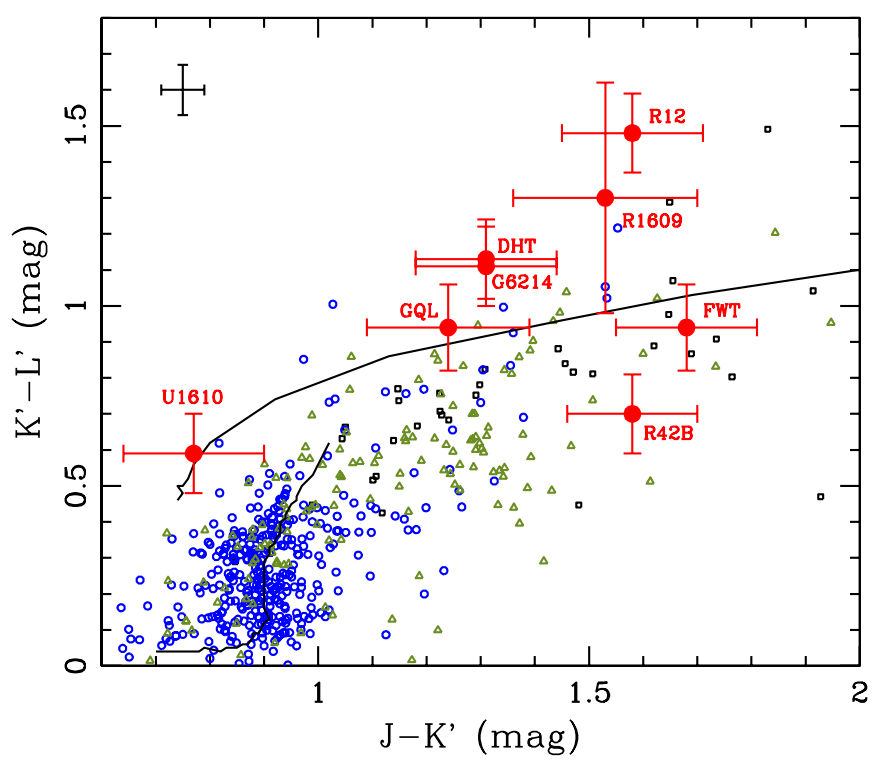

Figure 10. As in Figure 9, but showing dereddened $\left(J-K^{\prime}, K^{\prime}-L^{\prime}\right)$. Unlike for the marginal detection of an $H-K^{\prime}$ excess in Figure 9, there is a clear excess in $K^{\prime}-L^{\prime}$ for many PMCs compared with all of the reference populations of free-floating young objects.

(A color version of this figure is available in the online journal.)

interpreted the potentially red colors of GSC 6214-210 as a sign that it could host an optically thick disk; the existence of such a disk was confirmed by the discovery of ongoing accretion by Bowler et al. (2011). However, it is unclear whether five out of seven of the PMCs should be expected to host such a disk and 
observations of free-floating BDs of similar temperature and luminosity (Luhman et al. 2010) show that the excesses from optically thick disks are only evident at $\gtrsim 5 \mu \mathrm{m}$. Our reference population for Taurus includes many disk-bearing BDs (Luhman et al. 2010; Rebull et al. 2010), suggesting that the disks would need to be structurally different (i.e., with larger inner rim structures) to produce the observed colors.

We therefore suggest either that these objects host disks that are structurally different from those of free-floating BDs or that the red $K^{\prime}-L^{\prime}$ colors result from different atmospheric properties (i.e., dustier atmospheres or enhanced $\mathrm{H}_{2} \mathrm{O}$ absorption) than those of the free-floating population. The former explanation seems very plausible; since these PMCs generally have formed in the immediate neighborhood of a solar-type star, then they could have accreted a significantly more massive disk (perhaps approaching its Toomre stability limit) than a freefloating BD with a relatively anemic envelope. It is unlikely that these red colors denote the presence of lower-mass companions. Pairing of equal-mass (and equal-color) companions would only affect their positions in CMDs and based on the color-magnitude sequence of the DUSTY models, pairing of unequal-mass companions should move objects parallel to the color-color sequences, not perpendicular to them.

Further study at mid-infrared and submm $/ \mathrm{mm}$ wavelengths (to characterize the disk) and optical wavelengths (to characterize the accretion) should provide additional insight into the structure and evolution of these circumsubstellar (or circumplanetary) disks. Detailed analysis of the atmospheric properties $\left(T_{\text {eff }}, \log (g)\right.$, and $\left.[\mathrm{Fe} / \mathrm{H}]\right)$ will require spectroscopic followup (e.g., Lafrenière et al. 2008; Bowler et al. 2011) to directly compare the atomic lines and molecular bands with those of free-floating counterparts.

\section{SUMMARY}

We have discovered three PMCs in wide orbits around the young stars FW Tau (in Taurus) and ROXs 12 and ROXs 42B (in Ophiuchus). All three PMCs were reported as candidate companions in previous binary survey programs, but then were neglected for $>10 \mathrm{yr}$. We demonstrate with our own followup observations that each candidate is comoving with its host star. Based on the absolute $M_{K^{\prime}}$ magnitudes, we infer masses and projected physical separations of $10 \pm 4 M_{\mathrm{Jup}}$ and $330 \pm 30 \mathrm{AU}$ for FW Tau b, $16 \pm 4 M_{\text {Jup }}$ and $210 \pm 20 \mathrm{AU}$ for ROXs 12, and $10 \pm 4 M_{\text {Jup }}$ and $140 \pm 10 \mathrm{AU}$ for ROXs 42B b. We also have identified 10 other candidates to be unassociated field stars. Finally, we have obtained multicolor $J H K^{\prime} L^{\prime}$ NIR photometry for our three new companions and for five previously identified companions. The NIR photometry for our sample of eight known and new PMCs generally parallels the properties of free-floating low-mass BDs in these star-forming regions. However, five of the seven low-mass PMCs with $L^{\prime}$ photometry are redder in $K^{\prime}-L^{\prime}$ than free-floating counterparts of similar $J-K^{\prime}$. We speculate that this distinction could indicate a structural difference in the circumplanetary disks, perhaps tied to higher disk mass since at least two of the objects in our sample are known to be accreting vigorously.

We thank the referee for providing a thorough and helpful critique of this paper. A.L.K. was supported in part by a Clay Fellowship and by NASA through Hubble Fellowship grant 51257.01 awarded by STScI, which is operated by AURA, Inc., for NASA, under contract NAS 5-26555. M.J.I. acknowledges support from the Australian Research Council's Discovery program (DP1094977) and a Macquarie University Research Development Grant. T.D. was supported by NASA through Hubble Fellowship grant 51271.01 awarded by STScI, which is operated by AURA, Inc., for NASA, under contract NAS 5-26555. S.H. and L.C. were supported by NASA through the Sagan Fellowship program, and L.C. was supported by the ALMA-CONICYT fund allocated to the project number 31120009. M.L. acknowledges support from NSF grant AST0909222. Finally, this work was supported by a NASA Keck PI Data Award, administered by the NASA Exoplanet Science Institute. Some of the data presented herein were obtained at the W. M. Keck Observatory from telescope time allocated to NASA through the agency's scientific partnership with Caltech and the University of California. The Observatory was made possible by the generous financial support of the W. M. Keck Foundation.

The authors wish to recognize and acknowledge the very significant cultural role and reverence that the summit of Mauna Kea has always had within the indigenous Hawaiian community. We are most fortunate to have the opportunity to conduct observations from this mountain.

Note added in proof. The comovement of ROXs4213 b was also confirmed by Currie et al. (2014) based on an independent analysis of archival data including the 2011 observations we describe in Section 3.

\section{REFERENCES}

Ahn, C. P., Alexandroff, R., Allende Prieto, C., et al. 2012, ApJS, 203, 21 Allers, K. N., \& Liu, M. C. 2013, ApJ, 772, 79

Allers, K. N., Liu, M. C., Dupuy, T. J., \& Cushing, M. C. 2010, ApJ, 715, 561 Anderson, J., \& King, I. R. 2003, PASP, 115, 113

Andrews, S. M., \& Williams, J. P. 2005, ApJ, 631, 1134

Andrews, S. M., \& Williams, J. P. 2007, ApJ, 671, 1800

Bailey, V., Hinz, P. M., Currie, T., et al. 2013, ApJ, 767, 31

Baraffe, I., Chabrier, G., Allard, F., \& Hauschildt, P. H. 1998, A\&A, 337, 403

Baraffe, I., Chabrier, G., Barman, T. S., Allard, F., \& Hauschildt, P. H. 2003, A\&A, 402, 701

Bate, M. R. 2005, MNRAS, 363, 363

Biller, B. A., Liu, M. C., Wahhaj, Z., et al. 2013, ApJ, 777, 160

Bonnefoy, M., Chauvin, G., Rojo, P., et al. 2010, A\&A, 512, A52

Bouvier, J., \& Appenzeller, I. 1992, A\&AS, 92, 481

Bowler, B. P., Liu, M. C., Dupuy, T. J., \& Cushing, M. C. 2010, ApJ, 723, 850 Bowler, B. P., Liu, M. C., Kraus, A. L., Mann, A. W., \& Ireland, M. J. 2011, ApJ, 743,148

Bowler, B. P., Liu, M. C., Shkolnik, E. L., \& Dupuy, T. J. 2013, ApJ, 774, 55

Briceno, C., Calvet, N., Gomez, M., et al. 1993, PASP, 105, 686

Cameron, P. B. 2008, PhD thesis, California Institute of Technology

Carpenter, J. M. 2001, AJ, 121, 2851

Carpenter, J. M., Hillenbrand, L. A., Skrutskie, M. F., \& Meyer, M. R. 2002, AJ, 124,1001

Chabrier, G., Baraffe, I., Allard, F., \& Hauschildt, P. 2000, ApJ, 542, 464

Chauvin, G., Lagrange, A.-M., Bonavita, M., et al. 2010, A\&A, 509, A52

Chauvin, G., Lagrange, A., Dumas, C., et al. 2004, A\&A, 425, L29

Cruz, K. L., Kirkpatrick, J. D., \& Burgasser, A. J. 2009, AJ, 137, 3345

Currie, T., Daemgen, S., Debes, J., et al. 2014, ApJL, 780, L30

de Zeeuw, P. T., Hoogerwerf, R., de Bruijne, J. H. J., Brown, A. G. A., \& Blaauw, A. 1999, AJ, 117, 354

Delorme, P., Gagné, J., Girard, J. H., et al. 2013, A\&A, 553, L5

Dodson-Robinson, S. E., Veras, D., Ford, E. B., \& Beichman, C. A. 2009, ApJ, 707, 79

Duchêne, G., McCabe, C., Pinte, C., et al. 2010, ApJ, 712, 112

Evans, D. W., Irwin, M. J., \& Helmer, L. 2002, A\&A, 395, 347

Faherty, J. K., Rice, E. L., Cruz, K. L., Mamajek, E. E., \& Núñez, A. 2013, AJ, 145,2

Fortney, J. J., Marley, M. S., Saumon, D., \& Lodders, K. 2008, ApJ, 683, 1104 Galli, P. A. B., Bertout, C., Teixeira, R., \& Ducourant, C. 2013, A\&A, 558, A77 Ghez, A. M., Salim, S., Weinberg, N. N., et al. 2008, ApJ, 689, 1044

Hartmann, L., Calvet, N., Allen, L., Chen, H., \& Jayawardhana, R. 1999, AJ, 118,1784

Hayward, T. L., Brandl, B., Pirger, B., et al. 2001, PASP, 113, 105 
Hillenbrand, L. A., \& White, R. J. 2004, ApJ, 604, 741

Ireland, M. J., Kraus, A., Martinache, F., Law, N., \& Hillenbrand, L. A. 2011, ApJ, 726, 113

Itoh, Y., Hayashi, M., Tamura, M., et al. 2005, ApJ, 620, 984

Janson, M., Brandner, W., Henning, T., \& Zinnecker, H. 2006, A\&A, 453, 609

Jayawardhana, R., Ardila, D. R., Stelzer, B., \& Haisch, K. E., Jr. 2003, AJ, 126,1515

Kenyon, S. J., Dobrzycka, D., \& Hartmann, L. 1994, AJ, 108, 1872

Köhler, R., Kunkel, M., Leinert, C., \& Zinnecker, H. 2000, A\&A, 356, 541

Kratter, K. M., \& Murray-Clay, R. A. 2011, ApJ, 740, 1

Kratter, K. M., Murray-Clay, R. A., \& Youdin, A. N. 2010, ApJ, 710, 1375

Kraus, A. L., \& Hillenbrand, L. A. 2007a, ApJ, 662, 413

Kraus, A. L., \& Hillenbrand, L. A. 2007b, AJ, 134, 2340

Kraus, A. L., \& Hillenbrand, L. A. 2008, ApJL, 686, L111

Kraus, A. L., \& Hillenbrand, L. A. 2009a, ApJ, 704, 531

Kraus, A. L., \& Hillenbrand, L. A. 2009b, ApJ, 703, 1511

Kraus, A. L., \& Hillenbrand, L. A. 2012, ApJ, 757, 141

Kraus, A. L., Ireland, M. J., Hillenbrand, L. A., Martinache, F., \& Law, N. M. 2014, AJ, submitted

Kraus, A. L., Ireland, M. J., Martinache, F., \& Hillenbrand, L. A. 2011, ApJ, 731, 8

Kraus, A. L., Ireland, M. J., Martinache, F., \& Lloyd, J. P. 2008, ApJ, 679, 762

Kuzuhara, M., Tamura, M., Kudo, T., et al. 2013, ApJ, 774, 11

Lafrenière, D., Jayawardhana, R., \& van Kerkwijk, M. H. 2008, ApJL, 689, L153

Lafrenière, D., Jayawardhana, R., \& van Kerkwijk, M. H. 2010, ApJ, 719, 497

Leggett, S. K., Burningham, B., Saumon, D., et al. 2010, ApJ, 710, 1627

Lodieu, N., Hambly, N. C., Jameson, R. F., \& Hodgkin, S. T. 2008, MNRAS, 383, 1385

Loinard, L., Torres, R. M., Mioduszewski, A. J., \& Rodríguez, L. F. 2008, ApJL, 675, L29

Lombardi, M., Lada, C. J., \& Alves, J. 2008, A\&A, 489, 143

Luhman, K. L., Adame, L., D’Alessio, P., et al. 2007, ApJ, 666, 1219

Luhman, K. L., Allen, P. R., Espaillat, C., Hartmann, L., \& Calvet, N. 2010, ApJS, 186, 111

Luhman, K. L., Mamajek, E. E., Allen, P. R., \& Cruz, K. L. 2009, ApJ, 703,399

Luhman, K. L., Stauffer, J. R., Muench, A. A., et al. 2003, ApJ, 593, 1093

Luhman, K. L., Wilson, J. C., Brandner, W., et al. 2006, ApJ, 649, 894

Madhusudhan, N., Burrows, A., \& Currie, T. 2011, ApJ, 737, 34
Marleau, G., \& Cumming, A. 2013, MNRAS, 437, 1378

Marois, C., Macintosh, B., \& Barman, T. 2007, ApJ, 654, 151

Marois, C., Macintosh, B., Barman, T., et al. 2008, Sci, 322, 1348

Meru, F., \& Bate, M. R. 2010, MNRAS, 406, 2279

Metchev, S. A., \& Hillenbrand, L. A. 2009, ApJS, 181, 62

Monet, D. G., Levine, S. E., Canzian, B., et al. 2003, AJ, 125, 984

Neuhäuser, R., Guenther, E. W., Wuchterl, G., et al. 2005, A\&A, 435, L13

Neuhäuser, R., Mugrauer, M., Seifahrt, A., Schmidt, T. O. B., \& Vogt, N. 2008, A\&A, 484, 281

Nielsen, E. L., Liu, M. C., Wahhaj, Z., et al. 2013, ApJ, 776, 4

Patience, J., King, R. R., de Rosa, R. J., \& Marois, C. 2010, A\&A, 517, A76

Pecaut, M. J., Mamajek, E. E., \& Bubar, E. J. 2012, ApJ, 746, 154

Pollack, J. B., Hubickyj, O., Bodenheimer, P., et al. 1996, Icar, 124, 62

Rameau, J., Chauvin, G., Lagrange, A.-M., et al. 2012, A\&A, 546, A24

Rameau, J., Chauvin, G., Lagrange, A.-M., et al. 2013, ApJL, 772, L15

Ratzka, T., Köhler, R., \& Leinert, C. 2005, A\&A, 437, 611

Rebull, L. M., Padgett, D. L., McCabe, C.-E., et al. 2010, ApJS, 186, 259

Riaud, P., Mawet, D., Absil, O., et al. 2006, A\&A, 458, 317

Schlegel, D. J., Finkbeiner, D. P., \& Davis, M. 1998, ApJ, 500, 525

Schmidt, T. O. B., Neuhäuser, R., Seifahrt, A., et al. 2008, A\&A, 491, 311

Schmidt-Kaler, T. 1982, Landolt-Bornstein Numerical Data and Functional Relationships in Science and Technology, New Series, Group VI, Vol. 2b (Berlin: Springer)

Scholz, A., Jayawardhana, R., Wood, K., et al. 2008, ApJL, 681, L29

Seifahrt, A., Neuhäuser, R., \& Hauschildt, P. H. 2007, A\&A, 463, 309

Siess, L., Dufour, E., \& Forestini, M. 2000, A\&A, 358, 593

Simon, M., Ghez, A. M., Leinert, C., et al. 1995, ApJ, 443, 625

Skrutskie, M. F., Cutri, R. M., Stiening, R., et al. 2006, AJ, 131, 1163

Slesnick, C. L., Hillenbrand, L. A., \& Carpenter, J. M. 2008, ApJ, 688, 377

Spiegel, D. S., \& Burrows, A. 2012, ApJ, 745, 174

Stapelfeldt, K. R., Krist, J. E., Menard, F., et al. 1998, ApJL, 502, L65

Steinbring, E., Faber, S. M., Hinkley, S., et al. 2002, PASP, 114, 1267

Stetson, P. B. 1987, PASP, 99, 191

Torres, R. M., Loinard, L., Mioduszewski, A. J., \& Rodríguez, L. F. 2009, ApJ, 698, 242

Vigan, A., Patience, J., Marois, C., et al. 2012, A\&A, 544, A9

White, R. J., \& Ghez, A. M. 2001, ApJ, 556, 265

Wright, E. L., Eisenhardt, P. R. M., Mainzer, A. K., et al. 2010, AJ, 140, 1868

Yelda, S., Lu, J. R., Ghez, A. M., et al. 2010, ApJ, 725, 331

Zacharias, N. 2010, AJ, 139, 2208 